\title{
Traces of holomorphic families of operators on the noncommutative torus and on Hilbert modules
}

\author{
Sara Azzali, Cyril Lévy, Carolina Neira, Sylvie Paycha
}

February 28, 2022

\begin{abstract}
We revisit traces of holomorphic families of pseudodifferential operators on a closed manifold in view of geometric applications. We then transpose the corresponding analytic constructions to two different geometric frameworks; the noncommutative torus and Hilbert modules. These traces are meromorphic functions whose residues at the poles as well as the constant term of the Laurent expansion at zero (the latter when the family at zero is a differential operator) can be expressed in terms of Wodzicki residues and extended Wodzicki residues involving logarithmic operators. They are therefore local and contain geometric information. For holomorphic families leading to zeta regularised traces, they relate to the heat-kernel asymptotic coefficients via an inverse Mellin mapping theorem. We revisit Atiyah's $L^{2}$ index theorem by means of the (extended) Wodzicki residue and interpret the scalar curvature on the noncommutative two torus as an (extended) Wodzicki residue.
\end{abstract}

\section{Introduction}

The canonical trace $\operatorname{TR}(A(z))$ of a holomorphic family $\mathcal{A}: z \mapsto A(z)$ of classical pseudodifferential operators of affine order $-q z+a, q>0$ acting on smooth sections of a vector bundle over an $n$-dimensional closed manifold $(n>1)$, defines a meromorphic function $z \mapsto \operatorname{TR}(A(z))$ with a discrete set of simple poles $\left\{d_{j}=\frac{a+n-j}{q}, j \in \mathbb{Z}_{\geq 0}\right\}$. The residue at a pole $d_{j}$ is proportional to the Wodzicki residue of the operator $A\left(d_{j}\right)$. These are well-known results due to Wodzicki whose residue 39 is the only 1 (up to a multiplicative factor) trace on classical pseudodifferential operators of integer order, Guillemin [17] who introduced the notion of gauged symbols and Kontsevich and Vishik 20] whose canonical trace TR corresponds to the unique linear extension to operators of non integer order of the ordinary trace defined on operators of order $<-n$.

When $A(0)$ is a differential operator, there is no pole at zero since the residue vanishes on differential operators. It was later observed in 29] that the limit $\lim _{z \rightarrow 0} \operatorname{TR}(A(z))$ is also proportional to a Wodzicki residue, namely to the extended Wodzicki residue Res $\left(A^{\prime}(0)\right)$, extended since the operator $A^{\prime}(0)$ given by the derivative at zero is typically not a classical operator any longer. So the regularised trace $\lim _{z \rightarrow 0} \operatorname{TR}(A(z))$ is then local as a consequence of the locality of the Wodzicki residue.

\footnotetext{
${ }^{1}$ when the dimension of the manifold is greater than one.
} 
If the meromorphic map $\phi_{\mathcal{A}}: z \longmapsto \Gamma(z) \mathrm{TR}(A(z))$ corresponding to the holomorphic family $\mathcal{A}: z \longmapsto A(z)$ is the inverse Mellin transform of some function $f_{\mathcal{A}}: \mathbb{R}_{+} \longrightarrow \mathbb{R}$, it follows from the inverse Mellin mapping theorem (see Proposition 2.2) that $f_{\mathcal{A}}$ admits an asymptotic expansion at 0 given by

$$
f_{\mathcal{A}}(t)=\frac{1}{q} \sum_{j \geq 0} a_{j}(\mathcal{A}) t^{-d_{j}}+O\left(t^{-\gamma}\right),
$$

for some appropriate $\gamma$. Its singular coefficients are Wodzicki residues (Theorem 2.6)

$$
a_{j}(\mathcal{A})=-\frac{1}{q} \operatorname{Res}\left(A\left(d_{j}\right)\right) \quad \text { for } \quad d_{j}>0,
$$

and its constant term

$$
a_{j}(\mathcal{A})=-\frac{1}{q} \operatorname{Res}\left(A^{\prime}(0)\right) \quad \text { for } \quad d_{j}=0,
$$

is an extended Wodzicki residue.

For holomorphic families $\mathcal{A}: z \longmapsto A(z)=A Q^{-z}$ built from complex powers $Q^{-z}$ of some appropriate invertible elliptic operator $Q$ and a classical pseudodifferential operator $A$, the canonical trace $\operatorname{TR}(A(z))$ is called the $\zeta$-regularised trace $\zeta(A, Q)(z)$ of $A$ with respect to the weight $Q$. It follows from the above discussion, that if $A(0)=A$ is a differential operator, then $\zeta(A, Q)(0)$ is a local quantity proportional to the extended Wodzicki residue

$$
\operatorname{Res}(A \log Q)=-\frac{1}{q} \int_{M} \operatorname{res}_{x}(A \log Q) d x
$$

where $\operatorname{res}_{x}(A \log Q) d x$ is the pointwise extended residue density involving the logarithm of $Q$. When $A=I$ is the identity operator, this is the logarithmic residue $\operatorname{Res}(\log Q)$ investigated in 28, and 35.

When $Q=\Delta+\pi_{\Delta}$ with $\Delta$ an elliptic differential operator to which we add the orthogonal projection $\pi_{\Delta}$ onto its kernel, making $Q$ invertible, then $A(z)=$ $A Q^{-z}$ is the Mellin transform of the analytic family $\tilde{A}(t)=A e^{-t\left(\Delta+\pi_{\Delta}\right)}$. If $\Delta$ is nonnegative, the inverse Mellin transform of the meromorphic map $z \mapsto \zeta(A, Q)(z)$ is the Schwartz function

$$
f_{\mathcal{A}}: t \longmapsto \operatorname{Tr}\left(A e^{-t\left(\Delta+\pi_{\Delta}\right)}\right)=\int_{M} A K_{t}\left(\Delta+\pi_{\Delta}\right)(x, x) \sqrt{\operatorname{det}(g)}(x) d x
$$

on $] 0,+\infty$ [ where $K_{t}(A)(x, x)$ denotes the fibrewise trace at the point $x$ of the kernel of $e^{-t A}$ restricted to the diagonal and $\operatorname{det}(g)$ the determinant of a Riemannian metric $g$ on the manifold $M$.

When applied to any multiplication operator $A=\phi$ given by a smooth function $\phi$ on $M$, this shows that both the constant and the singular coefficients in the time zero asymptotic expansion of the kernel $K_{t}(\Delta)(x, x) 2$ can be expressed as

\footnotetext{
${ }^{2}$ It follows from Duhamel's formula [4 that the time zero asymptotic expansions of $K_{t}(\Delta+R)(x, x)$ and $K_{t}(\Delta)(x, x)$ coincide for every smoothing operator $R$ and hence in particular for $R=\pi_{\Delta}$, a fact that will be implicitly used throughout the paper.
} 
Wodzicki residues 3 (see Theorem 2.8):

$$
\begin{aligned}
& K_{t}(\Delta)(x, x) \sim_{t \rightarrow 0} \\
& -\frac{(4 \pi)^{\frac{n}{2}}}{2 \sqrt{\operatorname{det} g}(x)}\left[\operatorname{res}(\log \Delta) \delta_{\frac{n}{2}-\left[\frac{n}{2}\right]}\right. \\
& \left.+\sum_{k \in\left[0, \frac{n}{2}[\cap \mathbb{Z}\right.} \Gamma\left(\frac{n}{2}-k\right) \operatorname{res}_{x}\left(\Delta^{k-\frac{n}{2}}\right) t^{k-\frac{n}{2}}\right] .
\end{aligned}
$$

When integrated against $\phi \in C^{\infty}(M)$, the heat-kernel expansion (3) yields the following heat-operator trace expansion at zero

$$
\begin{aligned}
& \operatorname{Tr}\left(\phi e^{-t \Delta}\right) \sim_{t \rightarrow 0} \\
- & \frac{(4 \pi)^{\frac{n}{2}}}{2}\left[\operatorname{Res}(\phi \log \Delta) \delta_{\frac{n}{2}-\left[\frac{n}{2}\right]}\right. \\
+ & \left.\sum_{k \in\left[0, \frac{n}{2}[\cap \mathbb{Z}\right.} \Gamma\left(\frac{n}{2}-k\right) \operatorname{Res}\left(\phi \Delta^{k-\frac{n}{2}}\right) t^{k-\frac{n}{2}}\right] .
\end{aligned}
$$

That the singular coefficients of the heat-kernel (resp. heat-operator trace) expansion are proportional to the Wodzicki residue is a well-known (see e.g. [1, 19]) fact often held for folklore knowledge. It has been extended to noncommutative geometry [5, Formula 1.5]) for the asymptotic expansion of the spectral action (take $f(\lambda)=e^{-t \lambda}$ ) whose non-constant coefficients arise as Dixmier traces. What is lesser known, is that

- not only the singular coefficients in the heat-kernel expansion (resp. heatoperator trace) but also the constant coefficient are (possibly extended) Wodzicki residues (and hence local), a property that can be easily transposed to other geometric frameworks in which the canonical trace of holomorphic families of pseudodifferential operators can be built.

- no previous knowledge on the heat-operator trace asymptotics is needed to express its coefficients in terms of Wodzicki residues, which is a purely analytic procedure.

Since the Wodzicki residue of a logarithm is an algebraic expression involving the jets of the first $n$ homogeneous components of the symbol, we further recover other known facts (see [15. Lemma 1.8.2]), that the coefficients of the heat-operator trace expansion in (4) are

- functorial algebraic expressions in the jets of the homogeneous components of the symbol of the operator $\Delta$ as a consequence of the corresponding property of the Wodzicki residue.

- Consequently, if the operator $\Delta$ is of geometric nature, the coefficients are functorial algebraic expressions of the jets of the underlying metric and connection.

\footnotetext{
${ }^{3}$ Similarly, since the Wodzicki residue vanishes on smoothing operators, a perturbation of the operator by a smoothing operator so in particular by the projection onto the kernel does not modify the residue (see Corollary 1.9 ).
} 
Thanks to the functoriality of the construction, we can transpose our approach via inverse Mellin transforms to two different geometric contexts, applying it to

1. Holomorphic families of pseudodifferential operators on Hilbert modules: Let $\Delta$ be an (essentially) nonnegative selfadjoint differential operator acting on a vector bundle $E \rightarrow M$. One builds the operator $\Delta_{\mathcal{H}}$ by twisting $\Delta$ by a flat connection on a bundle $\mathcal{H}$ of Hilbert modules over a finite von Neumann algebra. One can then implement the same constructions as above to $\Delta_{\mathcal{H}}+R$ with $R$ a smoothing operator which makes the operator invertible (see Remark 3.14). The residue Res is then replaced by the $\tau$-residue $\operatorname{Res}^{\tau}$ (47), the $L^{2}$-trace (resp. canonical trace) $\operatorname{Tr}$ (resp. TR) by $\operatorname{Tr}^{\tau}$ (resp. $\operatorname{TR}^{\tau}$ ), where $\tau$ is a finite trace on the von Neumann algebra.

The corresponding heat-kernel $\tau$-trace $K_{t}^{\tau}\left(\Delta_{\mathcal{H}}\right)(x, x)$ at a point $x$ reads (see (63))

$$
\begin{array}{cl}
\sim_{t \rightarrow 0} & K_{t}^{\tau}\left(\Delta_{\mathcal{H}}\right)(x, x) \\
+ & \left.\sum_{k \in\left[0, \frac{n}{2}[\cap \mathbb{Z}\right.} \Gamma\left(\frac{n}{2}-k\right) \operatorname{res}_{x}^{\tau}\left(\Delta_{\mathcal{H}}^{k-\frac{n}{2}}\right) t^{k-\frac{n}{2}}\right] . \\
\sim_{t \rightarrow 0} & -\frac{(4 \pi)^{\frac{n}{2}}}{2 \sqrt{\operatorname{det} g}(x)}\left[\operatorname{res}_{x}^{\tau}\left(\log \Delta_{\mathcal{H}}\right) \delta_{\frac{n}{2}-\left[\frac{n}{2}\right]}(\log \Delta) \delta_{\frac{n}{2}-\left[\frac{n}{2}\right]}\right. \\
+ & \left.\sum_{k \in\left[0, \frac{n}{2}[\cap \mathbb{Z}\right.} \Gamma\left(\frac{n}{2}-k\right) \operatorname{res}_{x}\left(\Delta^{k-\frac{n}{2}}\right) t^{k-\frac{n}{2}}\right] .
\end{array}
$$

The above formula (5) follows from the following more general property (61) relating the (extended) residue of locally equivalent differential operators $A$, $A^{\prime}$, respectively $B, B^{\prime}$ (with some admissibility condition on the latter),

$$
\begin{gathered}
\operatorname{Res}^{\tau}(A \log B)=\operatorname{Res}^{\tau}\left(A^{\prime} \log B^{\prime}\right) \\
\operatorname{Res}^{\tau}\left(A B^{\alpha}\right)=\operatorname{Res}^{\tau}\left(A^{\prime} B^{\prime \alpha}\right), \quad \alpha \in \mathbb{R},
\end{gathered}
$$

where for simplicity we have dropped the projections onto the kernels since they are smoothing operators which are not "seen" by the residue. Atiyah's $L^{2}$-index theorem then boils down to an easy consequence, see Corollary 3.21 of the first of these two identities, using a $\mathbb{Z}_{2}$ graded version of the (extended) residue. This alternative proof, which is equivalent to Roe's heat equation proof [31, Ch.15], is of pseudodifferential analytic nature and relies on the locality of the Wodzicki residue.

2. Holomorphic families of pseudodifferential operators on the noncommutative torus: We consider a conformal perturbation $\boldsymbol{\Delta}_{h}$ (parameterised by a conformal factor $h$ ) of a Laplace-type operator $\boldsymbol{\Delta}$ acting on the noncommutative $n$-torus $\mathbb{T}_{\theta}^{n}$, where $\theta$ is an antisymmetric real matrix encoding the noncommutativity. The residue Res is then replaced by the $\theta$-residue $\operatorname{Res}_{\theta}$ (see [14), the $L^{2}$-trace (resp. canonical trace) $\operatorname{Tr}$ (resp. TR) by $\operatorname{Tr}_{\theta}$ (resp. $\mathrm{TR}_{\theta}$, see 24]). The coefficients of the Laurent expansions of traces of 
holomorphic families can be expressed in terms of Wodzicki residues (Theorem 4.1) and the heat-kernel expansion formula (44) reads for any $a \in \mathcal{A}_{\theta}$, the Fréchet algebra of "Schwartz functions" on the noncommutative torus $\mathbb{T}_{\theta}^{n}($ see $(\underline{68)})$

$$
\begin{aligned}
& \operatorname{Tr}_{\theta}\left(a e^{-t \boldsymbol{\Delta}_{h}}\right) \sim_{t \rightarrow 0} \\
&-\frac{(4 \pi)^{\frac{n}{2}}}{2}\left[\operatorname{Res} \theta\left(a \log \boldsymbol{\Delta}_{h}\right) \delta_{\frac{n}{2}-\left[\frac{n}{2}\right]}\right. \\
&\left.+\quad \sum_{k \in\left[0, \frac{n}{2}[\cap \mathbb{Z}\right.} \Gamma\left(\frac{n}{2}-k\right) \operatorname{Res}_{\theta}\left(a \Delta_{h}^{k-\frac{n}{2}}\right) t^{k-\frac{n}{2}}\right] .
\end{aligned}
$$

Going back to the setup of closed manifolds, for certain geometric operators $\Delta$, the coefficients of the heat-kernel expansion correspond to interesting geometric quantities; e.g. when $\Delta$ is the Laplace-Beltrami operator on a closed Riemannian manifold $(M, g)$, the coefficient of $t$ is proportional to the scalar curvature. The fact that the coefficient of $t$ in (6) provides an analogue in the noncommutative setup of the scalar curvature on a noncommutative torus was exploited in 6], 7], 8], 11, 12, 13, to compute a noncommutative analogue of the scalar curvature on noncommutative tori. By means of the Wodzicki residue on the noncommutative torus [14, we use (6) to define the scalar curvature $\mathfrak{s}_{h}$ as a Wodzicki residue; for any $a \in \mathcal{A}_{\theta}$ we set (compare with (69)

$$
\left\langle\mathfrak{s}_{h}, a\right\rangle_{h}= \begin{cases}-6 \pi \operatorname{Res}_{\theta}\left(a \log \boldsymbol{\Delta}_{h}\right) & \text { if } n=2 \\ -\frac{3}{2}(4 \pi)^{\frac{n}{2}} \Gamma\left(\frac{n}{2}-k\right) \operatorname{Res}_{\theta}\left(a \boldsymbol{\Delta}_{h}^{k-\frac{n}{2}}\right) & \text { otherwise }\end{cases}
$$

with $\langle\cdot, \cdot\rangle_{h}$ an adequate inner product on $\mathcal{A}_{\theta}$.

The extensions (5) and (6) are possible thanks to the fact that Cauchy calculus extends to Hilbert modules (see [3]) and to the noncommutative torus (see [14, 24]). The analogies between the pseudodifferential calculi in these two frameworks leads to the question whether the pseudodifferential calculus on $\mathcal{N} \mathbb{Z}^{n}$-Hilbert module encompasses the $\mathbb{Z}^{n}$-invariant pseudodifferential calculus on $\mathbb{R}^{n}$ studied in the more general context of global pseudodifferential calculus by Ruzhanski and Turunen in 33. The latter corresponds to the $\theta=0$ case of the algebra $\Psi\left(\mathbb{T}_{\theta}^{n}\right)$ described in Section 4, which raises the further question, namely whether this issue can be carried out to the noncommutative setup.

\section{Traces of holomorphic families on closed man- ifolds and their geometry}

For the purpose of a later generalisation to the Hilbert module setting, we recall the well-known basic setup to define the Wodzicki residue on closed manifolds and extend it to logarithmic pseudodifferential operators.

\subsection{The Wodzicki residue on pseudodifferential operators}

Let $(M, g)$ be a closed Riemannian manifold of dimension $n$, let $p: E \rightarrow M$ be a vector bundle and let $\operatorname{End}(E)=E^{*} \otimes E$ be the corresponding endomorphism 
bundle.

A linear operator $A: C^{\infty}(M, E) \rightarrow C^{\infty}(M, E)$ is a (classical) pseudodifferential operator of order $a \in \mathbb{C}$, denoted $A \in \Psi^{a}(M, E)$, if in some atlas of $E \rightarrow M$ it is of the form $A=\sum_{j=1}^{J} A_{j}+R$ where

- $R$ is a smoothing operator, namely a linear operator $R: C^{\infty}(M, E) \rightarrow$ $C^{\infty}(M, E)$ with Schwartz kernel given by a smooth section of the bundle $\mathcal{L}(M \times M, \operatorname{End}(E))$ of linear bounded operators whose fibre at $(x, y) \in M \times M$ is the Banach space of bounded linear operators from the fibre $E_{y}$ to the fibre $E_{x}$

- the operators $A_{j}$ are properly supported operators (meaning that the canonical projections $M \times M \rightarrow M$ restricted to the support of the Schwartz kernel are proper maps) from $C^{\infty}(M, E)$ into itself such that in any coordinate chart in a neighborhood $U$ of a point $x \in M$ the operators $A_{j}$ are of the form

$$
u \longmapsto \frac{1}{(2 \pi)^{n}} \int_{\mathbb{R}^{n}} d \xi \int_{U} d y e^{i\langle x-y, \xi\rangle} \sigma(x, \xi) u(y)
$$

for some symbol $\sigma \in C^{\infty}\left(U \times \mathbb{R}^{n}, \operatorname{End}(E)\right)$ which is asymptotically polyhomogeneous at infinity

$$
\sigma(x, \xi) \sim \sum_{i=0}^{\infty} \omega(\xi) \sigma_{a-i}(x, \xi)
$$

i.e., the components $\sigma_{a-i} \in C^{\infty}\left(T^{*} U \backslash(U \times\{0\})\right.$, $\left.\operatorname{End}(E)\right)$ being positively homogeneous of degree $a-i$ with $a$ the order of the operator. The asymptotic behaviour is to be understood as 4

$$
\sigma^{(N)}(x, \xi):=\sigma(x, \xi)-\sum_{i=0}^{N-1} \omega(\xi) \sigma_{a-i}(x, \xi)
$$

is a symbol of order $\Re(a)-N$ for any $N \in \mathbb{N}$. Also, $\omega$ is a smooth function on $\mathbb{R}^{n}$ which is zero in a neighborhood of zero and identically one outside the unit ball.

Let us denote by $\mathcal{S}^{a}(U, \operatorname{End}(E))$ the set of such symbols and by

$$
\mathcal{S}(U, \operatorname{End}(E))=\left\langle\cup_{a \in \mathbb{C}} \mathcal{S}^{a}(U, \operatorname{End}(E))\right\rangle
$$

the algebra generated by all classical symbols of any complex order. Finally, let us denote by $\Theta(M, E)$ the subalgebra in $\Psi(M, E)$ of differential operators.

Let $\operatorname{tr}_{x}$ denote the fibrewise trace on $\operatorname{End}(E)$ above a point $x \in M$. The pointwise Wodzicki residue of the operator $A$ with symbol $\sigma(A)$ at the point $x$ defined as

$$
\operatorname{res}_{x}(A):=\int_{|\xi|_{x}=1} \operatorname{tr}_{x}\left(\sigma(A)_{-n}(x, \xi)\right) d_{S} \xi
$$

vanishes on smoothing symbols so that it is independent of the choice of cut-off function $\omega$ chosen in (8). Here $d_{S} \xi$ is the measure on the unit cotangent sphere $S_{x}^{*} M=\left\{\xi \in T_{x}^{*} M,|\xi|_{x}:=g_{x}(\xi, \xi)=1\right\}$ induced by the one on the cotangent space $T_{x}^{*} M$ to $M$ at the point $x$ given by a Riemannian metric $g$ on $M, d_{S}$ denotes the corresponding normalised measure $d_{S} \xi=\frac{1}{(2 \pi)^{n}} d_{S} \xi$.

\footnotetext{
${ }^{4}$ The remainder depends on the choice of the cut-off function $\omega$ which is not explicitly mentioned here to alleviate the notation.
} 
Remark 1.1. Clearly the residue res $x_{x}$ vanishes on differential and non integer order operators.

An important result of Wodzicki is that $\operatorname{res}_{x}(A) d x$ defines a global density [39]. The Wodzicki residue of the operator $A$ is then defined by

$$
\operatorname{Res}(A)=\int_{M} \operatorname{res}_{x}(A) d x=\int_{M} d x \int_{|\xi|_{x}=1} \operatorname{tr}_{x}\left(\sigma_{-n}(A)(x, \xi)\right) d_{S} \xi .
$$

\subsection{Logarithms of pseudodifferential operators on closed man- ifolds}

Definition 1.2. (see e.g. 37, 36) Let $A$ be an operator in $\Psi(M, E)$. A real number $\beta$ is a principal angle of $A$ if there exists a ray $R_{\beta}=\left\{r e^{i \beta}, \quad r \geq 0\right\}$ which is disjoint from the spectrum of the $\operatorname{End}\left(E_{x}\right)$-valued leading symbol $\sigma_{L}(A)(x, \xi)$ for any $x \in M, \xi \in T_{x}^{*} M \backslash\{0\}$.

Definition 1.3. We call an operator $A \in \Psi(M, E)$ admissible with spectral cut $\beta$ if

- its order is positive,

- $\beta$ is a principal angle for $A$.

Remark 1.4. An admissible operator is elliptic but not necessarily invertible. Admissibility is a covariant condition, that is to say it is preserved under diffeomorphisms.

Definition 1.5. We call weight an invertible admissible operator $Q \in \Psi(M, E)$.

As we shall see later, weights are used to regularise traces. Here is a useful lemma which strongly uses the theory of elliptic operators on closed manifolds.

Lemma 1.6. If $A \in \Psi(M, E)$ is an admissible operator with spectral cut $\beta$ then there is an angle $\beta^{\prime}$ arbitrarily close if not equal to $\beta$ and a truncated solid angle $\Lambda_{\beta^{\prime}, \epsilon}:=\left\{z \in \mathbb{C} \backslash 0:|z|>\epsilon, \arg z \in\left(\beta^{\prime}-\epsilon, \beta^{\prime}+\epsilon\right)\right\}$ for some $\epsilon>0$, outside of which lies the spectrum of $A$.

If $A$ is moreover invertible i.e., if it is a weight, then its spectrum lies outside the solid angle $V_{\beta^{\prime}, \epsilon}:=\left\{z \in \mathbb{C} \backslash 0: \arg z \in\left(\beta^{\prime}-\epsilon, \beta^{\prime}+\epsilon\right)\right\}$ for some small $\epsilon>0$. The angle $\beta^{\prime}$ is then called an Agmon angle.

Proof. Since $A$ is admissible, it is elliptic. Since the order of $A$ is positive, the manifold $M$ being closed, the operator has a purely discrete spectrum, which consists of countably many eigenvalues with no accumulation point. So if the spectrum of $A$ meets the ray $R_{\beta}$, there is a small perturbation $\beta^{\prime}$ of $\beta$ such that the spectrum of $A$ does not meet $R_{\beta^{\prime}} \backslash\{0\}$. Being discrete, the spectrum of $A$ actually lies outside a truncated solid angle $\left\{z \in \mathbb{C} \backslash 0:|z|>\epsilon, \arg z \in\left(\beta^{\prime}-\epsilon, \beta^{\prime}+\epsilon\right)\right\}$ for some $\epsilon>0$ chosen small enough so that 0 is the only eigenvalue in the ball of radius $\epsilon$ centered at zero. If moreover $A$ is invertible, then its spectrum lies outside the solid angle $\left\{z \in \mathbb{C} \backslash 0: \arg z \in\left(\beta^{\prime}-\epsilon, \beta^{\prime}+\epsilon\right)\right\}$ and $\beta^{\prime}$ is an Agmon angle for A.

Let now $A$ be a weight in $\Psi^{a}(M, E)$ with spectral cut $\beta$. Then for $\Re(z)>0$, its complex powers (see [37] for further details)

$$
A_{\beta}^{z}=\frac{i}{2 \pi} \int_{\Gamma_{\beta}} \lambda^{z}(\lambda-A)^{-1} d \lambda,
$$


and respectively the operators [36, Par. 2.6.1.2.]

$$
L_{\beta}(A, z)=\frac{i}{2 \pi} \int_{\Gamma_{\beta}} \log _{\beta} \lambda \lambda^{z}(\lambda-A)^{-1} d \lambda,
$$

are bounded linear maps from any Sobolev closure $H^{s}(M, E)$ of $C^{\infty}(M, E), s \in \mathbb{R}$, with values in $H^{s-a \Re(z)}(M, E)$ respectively in $H^{s-a \Re(z)+\epsilon}(M, E)$, for any $\epsilon>0$. Here $\Gamma_{\beta}$ is a closed contour in $\mathbb{C} \backslash\left\{r e^{i \beta}, r \geq 0\right\}$ around the spectrum of $A$ oriented clockwise. These definitions extend to the whole complex plane

$$
A_{\beta}^{z}=A^{k} A_{\beta}^{z-k}, \text { resp. } L_{\beta}(A, z)=A^{k} L_{\beta}(A, z-k), \quad \Re(z)<k
$$

for any $k \in \mathbb{N}, A_{\beta}^{z}$ is an operator in $\Psi(M, E)$ of order $a z$ for any complex number $z$, and the logarithm

$$
\log _{\beta}(A):=L_{\beta}(A, 0)
$$

of $A$ is a bounded linear map from $H^{s}(M, E)$ to $H^{s+\epsilon}(M, E), \forall \epsilon>0$. One has by construction $\log _{\beta} A A_{\beta}^{z}=A_{\beta}^{z} \log _{\beta} A, \forall z \in \mathbb{C}$.

Remark 1.7. Just as a complex power does, the logarithm depends on the choice of a spectral cut $\beta$. However, in order to simplify the notation we shall often drop the explicit mention of $\beta$.

The logarithm of a classical pseudodifferential operator of positive order is not classical. Indeed, in a local trivialisation, the symbol of $\log _{\beta} A$ reads (see e.g. 36])

$$
\sigma\left(\log _{\beta} A\right)(x, \xi)=a \log |\xi| I+\sigma_{\mathrm{cl}}\left(\log _{\beta} A\right)(x, \xi)
$$

where $a$ denotes the order of $A$ and $\sigma_{\mathrm{cl}}\left(\log _{\beta} A\right)$ is a classical symbol of order zero with homogeneous components $\sigma_{-j}\left(\log _{\beta} A\right)$ of degree $-j, j \in \mathbb{Z}_{\geq 0}$.

Moreover, the leading symbol $\sigma_{\mathrm{cl}}^{L}\left(\log _{\beta} A\right)$ of $\sigma_{\mathrm{cl}}\left(\log _{\beta} A\right)$ can be expressed in terms of the leading symbol $\sigma^{L}(A)$ of $A$ as

$$
\sigma_{\mathrm{cl}}^{L}\left(\log _{\beta} A\right)(x, \xi)=\log _{\beta}\left(\sigma^{L}(A)\left(x, \frac{\xi}{|\xi|}\right)\right) \quad \forall(x, \xi) \in T^{*} M \backslash M \times\{0\} .
$$

\subsection{The local Wodzicki residue extended to logarithms}

In spite of the fact that logarithms are not classical, the Wodzicki residue on classical pseudodifferential operators does extend to logarithms 5 As a first step we extend to logarithms the local residue res $_{x}$ defined in (10). For a weight $Q \in$ $\Psi(M, E)$ with spectral cut $\beta$ we define the pointwise extended residue as

$$
\operatorname{res}_{x}\left(\log _{\beta} Q\right):=\int_{|\xi|_{x}=1} \operatorname{tr}_{x}\left(\sigma_{-n}\left(\log _{\beta} Q\right)(x, \xi)\right) d_{S} \xi .
$$

By (14) this is a natural extension of the pointwise residue on classical symbols since the integral over the sphere vanishes on the logarithm of the norm. The fact that the $n$-form $\operatorname{res}_{x}\left(\log _{\beta} Q\right) d x$ defines a volume density will arise later as a consequence of a local formula for the $\zeta$-regularised trace.

The subsequent proposition shows the locality of the extended residue of $A \log Q$ in so far as it only depends on a finite number of homogeneous components of the symbols of $A$ and $Q$

\footnotetext{
${ }^{5}$ This extended residue differs from the higher residue on log-polyhomogeneous operators introduced in 21 .
} 
Proposition 1.8. For any weight $Q \in \Psi(M, E)$ of order $q$,

1. (compare with [15, Lemma 1.8.2])

the local logarithmic residue at a point $x \in M$ which reads

$$
\operatorname{res}_{x}(\log Q)=\frac{1}{2 \pi i} \int_{|\xi|_{x}=1}\left(\int_{\Gamma} \log \lambda \sigma_{-q-n}(Q-\lambda)^{-1}(x, \xi) d \lambda\right) d_{S} \xi
$$

where $\Gamma$ is a contour around the spectrum of $Q$ oriented clockwise, is an algebraic expression in the $x$-jets of the first $n$ homogeneous components (taken in decreasing order of homogeneity) of the symbol $\sigma(Q)(x, \cdot)$ of $Q$ at that point given by the integral over the unit cotangent sphere of an algebraic expression in the $(x, \xi)$-jets of the first $n$ homogeneous components of the symbol $\sigma(Q)(x, \cdot)$ of $Q$ at that point. Here $\sigma_{-q-n}(Q-\lambda)^{-1}$ is the $(-q-n)$ th homogeneous component of the resolvent $(Q-\lambda)^{-1}$ of $Q$.

2. (compare with [15, Lemma 1.9.1])

Given a differential operator $A=\sum_{|\alpha| \leq a} a_{\alpha}(x) D_{x}^{\alpha} \in \Theta(M, E)$ of order a $\in$ $\mathbb{Z}_{\geq 0}$, then $\operatorname{res}_{x}(A \log Q)$ is an algebraic expression in the coefficients $a_{\alpha}$ of $A$ and in the $x$-jets of the first $n+a$ homogeneous components of the symbol $\sigma(Q)(x, \cdot)$ of $Q$ at that point.

In particular, if $Q=\sum_{|\beta| \leq q} b_{\beta}(x) D_{x}^{\beta}$ is a differential operator, then the local residue $\operatorname{res}_{x}(A \log (Q))$ is an algebraic expression in the coefficients $a_{\alpha}$ and in the $x$-jets of the coefficients $b_{\beta}$.

Proof. Since

$$
\begin{aligned}
\sigma_{-n}(A \log Q) & =\sum_{|\gamma|+j+k=a+n} \frac{(-i)^{\gamma}}{\gamma !} \partial_{\xi}^{\gamma} \sigma_{a-j}(A) \partial_{x}^{\gamma} \sigma_{-k}(\log Q) \\
& =\sum_{|\gamma|+k=a+n}(-i)^{\gamma}\left(\begin{array}{l}
\alpha \\
\gamma
\end{array}\right) a_{\alpha}(x) \xi^{a-\gamma} \partial_{x}^{\gamma} \sigma_{-k}(\log Q),
\end{aligned}
$$

the fact that $\operatorname{res}_{x}(A \log Q)$ is an algebraic expression in the coefficients $a_{\alpha}$ of $A$ and in the $x$-jets of the first $n+a$ homogeneous components of the symbol $\sigma(Q)(x, \cdot)$ follows from a similar statement for the homogeneous components $\sigma_{-k}(\log Q)$ of order $-k \in \mathbb{Z}_{\leq 0}$ of the symbol of $\log Q$. Now, since $\log Q=\left.\partial_{z} Q^{z}\right|_{z=0}$, the homogeneous component $\sigma_{-k}(\log Q)$ is derived by differentiating the homogeneous component $\sigma_{q z-k}\left(Q^{z}\right)$ of degree $q z-k$ at zero of the complex power $Q^{z}$. The latter is obtained from the homogeneous component $\sigma_{-q-k}$ of the resolvent $(Q-\lambda)^{-1}$ by means of the Cauchy formula

$$
Q^{z}=\frac{1}{2 i \pi} \int_{\Gamma} \lambda^{z}(Q-\lambda)^{-1} d \lambda
$$

where, as before, $\Gamma$ is a contour around the spectrum of $Q$ oriented clockwise. Thus we find

$$
\sigma_{-k}(\log Q)=\frac{1}{2 i \pi} \partial_{z}\left(\int_{\Gamma} \lambda^{z} \sigma_{-q-k}(Q-\lambda)^{-1} d \lambda\right)_{\left.\right|_{z=0}} .
$$

In particular, setting $k=n$ and integrating over the unit cotangent sphere we find

$$
\operatorname{res}_{x}(\log Q)=\frac{1}{2 \pi i} \int_{|\xi|_{x}=1} \partial_{z}\left(\int_{\Gamma} \lambda^{z} \sigma_{-q-n}(Q-\lambda)^{-1} d \lambda\right)_{\left.\right|_{z=0}} d_{S} \xi
$$


which yields (16). Since $\sigma_{-q-k}(Q-\lambda)^{-1}(x, \xi)$ is an algebraic expression in the $(x, \xi)$-jets of the first $k$ homogeneous components

$$
\sigma_{q-1}(Q)(x, \xi), \sigma_{q-2}(Q)(x, \xi), \cdots, \sigma_{q-k}(Q)(x, \xi)
$$

of the symbol of $Q$, so is $\sigma_{-k}(\log Q)(x, \xi)$ an algebraic expression in the jets of the first $k$ homogeneous components. This for $n=k$ yields the first assertion (compare with Formula (8) in 25). The second assertion follows in a similar way after implementing the differential operator $A$ and integrating over the unit cotangent sphere.

The locality of the extended residue can also be seen from the fact that it does not detect smoothing perturbations.

Corollary 1.9. Let $A \in \Psi(M, E)$ be an admissible operator. Let $R, S$ be two smoothing operators acting on $C^{\infty}(M, E)$ such that the perturbed operators $A+R$ and $A+S$ are invertible. They define weights and for any $x \in M$ we have

$$
\operatorname{res}_{x}(\log (A+R))=\operatorname{res}_{x}(\log (A+S)) .
$$

Proof. This follows from Proposition 1.8 and the fact that a smoothing perturbation of an operator does not modify the homogeneous components of its symbol.

Let $\Delta \in \Theta(M, E)$ be an admissible operator. Let $E$ be equipped with a hermitian metric, which combined with a Riemannian metric on $M$ induces an inner product on $C^{\infty}(M, E)$. It follows from the theory of elliptic operators on a closed manifold (see e.g. [15) that the orthogonal projection $\pi_{\Delta}$ onto the kernel Ker $(\Delta)$ is a finite rank operator and hence smoothing. Consequently, the operator $Q:=\Delta+\pi_{\Delta}$ is a weight. On the grounds of Corollary [1.9, we define the pointwise logarithmic residue of $\Delta$ as

$$
\operatorname{res}_{x}(\log \Delta):=\operatorname{res}_{x}\left(\log \left(\Delta+\pi_{\Delta}\right)\right) \quad \forall x \in M .
$$

As we shall see below, the local density $\operatorname{res}_{x}(A \log \Delta) d x$ actually defines a global density on the manifold, confirming the known fact that the residue extends to logarithms [27, 28, 29].

\subsection{The Wodzicki residue as a complex residue}

Given a symbol $\sigma(x, \xi) \in \mathcal{S}^{a}(U, \operatorname{End}(E))$ with $x$ a point in $M$ and $U \subset M$ an open neighborhood of $x$, the cut-off integral 6 is defined as the finite part

$$
f_{\mathbb{R}^{n}} \operatorname{tr}_{x} \sigma(x, \xi) d \xi:=\mathrm{fp}_{R \rightarrow \infty} \int_{B(0, R)} \operatorname{tr}_{x} \sigma(x, \xi) d \xi
$$

Remark 1.10. Whereas the residue vanishes on symbols whose order has real part smaller than $-n$, the cut-off integral coincides on those symbols with the ordinary integral on $\mathbb{R}^{n}$. A straightforward computation shows that, like the local residue, the cut-off integral also vanishes on polynomial symbols.

\footnotetext{
16.

${ }^{6}$ also called Hadamard finite part integral see e.g. [36. Example 2 chapter II], and also
} 
We need holomorphic families of classical pseudodifferential symbols first introduced by Guillemin in 17] and extensively used by Kontsevich and Vishik in 20. The idea is to embed a symbol $\sigma$ in a family $z \mapsto \sigma(z)$ depending holomorphically on a complex parameter $z$.

Definition 1.11. Let $U$ be an open subset of $M$. We call a family $(\sigma(z))_{z \in \Omega}$ of symbols in $\mathcal{S}(U, \operatorname{End}(E))$ parametrised by a domain $\Omega$ of $\mathbb{C}$ holomorphic at a point $z_{0} \in \Omega$ if, with the notation of (8) and (9) we have

1. $\sigma(z)(x, \cdot)$ is uniformly in $x$ on any compact subset of $U$, holomorphic at $z_{0}$ as a function of $z$ with values in $C^{\infty}\left(U \times \mathbb{R}^{n}, \operatorname{End}(E)\right)$,

2. for any $z$ in a neighborhood of $z_{0}$ there is an asymptotic expansion of the type (8)

$$
\sigma(z)(x, \cdot) \sim \sum_{j \geq 0} \sigma_{\alpha(z)-j}(z)(x, \cdot),
$$

with $\alpha(z):=-q z+a$ for some positive number $q$ and $a$ the order of $\sigma:=\sigma(0)$,

3. for any integer $N \geq 1$ the remainder

$$
\sigma_{(N)}(z):=\sigma(z)-\sum_{j=0}^{N-1} \sigma_{\alpha(z)-j}(z)
$$

is uniformly in $x$ on any compact subset of $U$, holomorphic at $z_{0}$ as a function of $z$ with values in $C^{\infty}\left(U \times \mathbb{R}^{n}\right.$, $\left.\operatorname{End}(E)\right)$ with $k$-th $z$-derivative

$$
\sigma_{(N)}^{(k)}(z):=\partial_{z}^{k}\left(\sigma_{(N)}(z)\right)
$$

a symbol on $U$ of order $\alpha(z)-N+\epsilon$ for any $\epsilon>0$ uniformly in $x$ on any compact subset of $U$ and locally uniformly in $z$ around $z_{0}$, i.e. the $k$-th derivative $\partial_{z}^{k} \sigma_{(N)}(z)$ satisfies a local uniform estimate in $z$ around $z_{0}$

$$
\left\|\partial_{\xi}^{\beta} \partial_{z}^{k} \sigma_{(N)}(z)(x, \xi)\right\| \leq C_{\beta}\langle\xi\rangle^{\Re(q z)-N-|\beta|} \quad \forall \xi \in \mathbb{R}^{n},
$$

where $\|A\|:=\sqrt{\operatorname{tr}_{x}\left(A^{*} A\right)}$ is the norm on $\mathcal{L}(M \times M$, End $(E))$ and where we have set $\langle\xi\rangle:=\sqrt{1+|\xi|^{2}}$ with $|\cdot|$ the Euclidean norm of $\xi$.

Example 1.12. If $\sigma \in S(U, \operatorname{End}(E))$ is a symbol of order $\alpha(0)$, then $\sigma(z)(x, \xi)=$ $\sigma(x, \xi)\langle\xi\rangle^{-z}$ defines a holomorphic family of order $\alpha(z)=-z+\alpha(0)$.

The following assertion can be shown on direct inspection of the cut-off integral.

Proposition 1.13. For any holomorphic family $\sigma(z)$ of classical symbols parametrised by $\mathbb{C}$ with affine order $\alpha(z)=-q z+$ a for some positive real number $q$ and some real number a,

1. the map

$$
z \mapsto f_{\mathbb{R}^{n}} \sigma(z)(x, \xi) d \xi
$$

is meromorphic with simple poles $d_{j}:=\frac{a+n-j}{q}, \quad j \in \mathbb{Z}_{\geq 0}$. 
2. 20] The complex residue at the point $d_{j}$ in $\mathbb{C}$ is given by:

$$
\operatorname{Res}_{z=d_{j}}\left(f_{\mathbb{R}^{n}} \operatorname{tr}_{x} \sigma(z)(x, \xi) d \xi\right)=\frac{1}{q} \operatorname{res}_{x}\left(\sigma\left(d_{j}\right)\right) .
$$

3. 29] The finite part at the pole $d_{j}$ differs from the cut-off regularised integral $f_{\mathbb{R}^{n}} \operatorname{tr}_{x} \sigma\left(d_{j}\right)(x, \xi) d \xi$ by

$$
\mathrm{fp}_{z=d_{j}}\left(f_{\mathbb{R}^{n}} \operatorname{tr}_{x} \sigma(z)(x, \xi) d \xi\right)-f_{\mathbb{R}^{n}} \operatorname{tr}_{x} \sigma\left(d_{j}\right)(x, \xi) d \xi=\frac{1}{q} \operatorname{res}_{x}\left(\sigma^{\prime}\left(d_{j}\right)\right)
$$

Here the noncommutative residue is extended to the possibly non-classical symbol $7 \tau_{j}(x, \xi):=\sigma^{\prime}\left(d_{j}\right)(x, \xi)$ using the same formula as in Equation (10)

$$
\operatorname{res}_{x}\left(\tau_{j}\right):=\int_{\left.|\xi|\right|_{x}=1} \operatorname{tr}_{x}\left(\tau_{j}\right)_{-n}(x, \xi) d_{S} \xi
$$

We are now ready to introduce holomorphic families of pseudodifferential operators.

Definition 1.14. Following [29, Definition 1.14] we call a family $(A(z))_{z \in \Omega}$ of operators in $\Psi(M, E)$ parametrised by a domain $\Omega$ of $\mathbb{C}$ holomorphic at a point $z_{0} \in \Omega$ if, with the notation of Section 1.1, in each local trivialisation $U$ of $E \rightarrow M$ we have

$$
A(z)=\sum_{j=1}^{J} A_{j}(z)+R(z)
$$

with

1. $A_{j}(z)=\operatorname{Op}\left(\sigma_{j}(z)\right)$, where $\sigma_{j}(z)$ is a holomorphic family of polyhomogeneous symbols on $U$,

2. $R(z)$ is a smoothing operator with Schwartz kernel $R(z, x, y) \in C^{\infty}(\Omega \times U \times$ $U, \operatorname{End}(E))$ holomorphic in $z$.

Integrating the results of Proposition 1.13 over $M$ yields the following theorem which we quote without proof, referring the reader to [20] and 29]. Let us however recall that the linear map TR introduced in the theorem below is the canonical trace popularised in [20] i.e., the unique linear form (up to a multiplicative factor) on the subset of $\Psi(M, E)$ consisting of non integer order classical pseudodifferential operators, which vanishes on commutators that lie in this set. It extends to differential operators where it vanishes and it coincides with the $L^{2}$-trace $\operatorname{Tr}$ on trace-class operators i.e. the real part of the order is smaller than $-n$.

Theorem 1.15. For any holomorphic family $A(z) \in \Psi(M, E)$ of classical operators parametrised by $\mathbb{C}$ with holomorphic order $-q z+a$ for some positive $q$ and some real number a,

1. the meromorphic map $z \mapsto \operatorname{TR}_{x}(A(z)):=f_{\mathbb{R}^{n}} \operatorname{tr}_{x} \sigma(z)(x, \xi) d \xi$ integrates over $M$ to the map

$$
z \mapsto \operatorname{TR}(A(z)):=\int_{M} \operatorname{TR}_{x}(A(z)) d x
$$

which is meromorphic with simple poles $d_{j}:=\frac{a+n-j}{q}, \quad j \in \mathbb{Z}_{\geq 0}$.

\footnotetext{
${ }^{7}$ The asymptotic expansion of $\tau_{j}(x, \xi)$ as $|\xi| \rightarrow \infty$ might present logarithmic terms $\log |\xi|$, which vanish on the unit sphere and therefore do not explicitly arise in the following definition.
} 
2. 20] The complex residue at the point $d_{j}$ is given by:

$$
\operatorname{Res}_{z=d_{j}} \operatorname{TR}(A(z))=\frac{1}{q} \operatorname{Res}\left(A\left(d_{j}\right)\right) .
$$

3. 29] If $A\left(d_{j}\right)$ differs from a differential operator 8 by a trace-class pseudodifferential operator $T_{j}$, then $A\left(d_{j}\right)$ has a well-defined canonical trace $\operatorname{TR}\left(A\left(d_{j}\right)\right)=$ $\operatorname{Tr}\left(T_{j}\right)$ and $A^{\prime}\left(d_{j}\right)$ has a well defined Wodzicki residue

$$
\operatorname{Res}\left(A^{\prime}\left(d_{j}\right)\right):=\int_{M} \operatorname{res}_{x}\left(A^{\prime}\left(d_{j}\right)\right) d x
$$

where

$$
\operatorname{res}_{x}\left(A^{\prime}\left(d_{j}\right)\right):=\int_{|\xi|_{x}=1} \sigma_{-n}\left(A^{\prime}\left(d_{j}\right)\right)(x, \xi) d_{S} \xi
$$

at the point $d_{j}$ and we have

$$
\mathrm{fp}_{z=d_{j}} \operatorname{TR}(A(z))=\operatorname{Tr}\left(T_{j}\right)-\frac{1}{q} \operatorname{Res}\left(A^{\prime}\left(d_{j}\right)\right) .
$$

Remark 1.16. Formula (26) formally follows from (24) applied to the family $\tau_{j}(z)=\frac{\sigma(z)-\sigma\left(d_{j}\right)}{z-d_{j}}$ since $\tau_{j}\left(d_{j}\right)=\sigma^{\prime}\left(d_{j}\right)$. However, $\tau_{j}(z)$ not strictly speaking being a holomorphic family of classical symbols since the two symbols $\sigma(z)$ and $\sigma\left(d_{j}\right)$ have different orders outside $d_{j}$, which do not differ by an integer, the proof is actually slightly more indirect.

Consequently, $\operatorname{Res}\left(A^{\prime}\left(d_{j}\right)\right)$ is the noncommutative residue extended to the typically non-classical operator $A^{\prime}\left(d_{j}\right)$.

\section{$1.5 \zeta$-regularised traces}

Given a weight $Q \in \Psi(M, E)$ of order $q \in \mathbb{R}_{+}$and an operator $A \in \Psi(M, E)$ (not necessarily admissible) of order $a \in \mathbb{R}$, the map $z \mapsto A(z):=A Q^{-z}$ defines a holomorphic family. The subsequent theorem quoted from [29] follows from applying Theorem 1.15 to this family.

Theorem 1.17. Given a weight $Q \in \Psi(M, E)$ of order $q \in \mathbb{R}_{+}$and an operator $A \in \Psi^{a}(M, E)$, the map

$$
z \mapsto \zeta(A, Q)(z):=\operatorname{TR}\left(A Q^{-z}\right)
$$

called the $\zeta$-regularised trace of $A$ with respect to the weight $Q$, is holomorphic on a half plane $\Re(z)>\frac{n+a}{q}$, meromorphic on the whole complex plane with poles at $d_{j}=\frac{a+n-j}{q}, j \in \mathbb{Z}_{\geq 0}$ and the complex residue at this pole can be expressed as a Wodzicki residue

$$
\operatorname{Res}_{z=d_{j}} \zeta(A, Q)(z)=\frac{1}{q} \operatorname{Res}\left(A Q^{-d_{j}}\right) .
$$

If $A$ differs from a differential operator by a trace-class pseudodifferential operator $T$, the $n$-form

$$
\operatorname{res}_{x}(A \log Q) d x:=\int_{|\xi|_{x}=1} \sigma_{-n}(A \log Q)(x, \xi) d_{S} \xi d x .
$$

\footnotetext{
${ }^{8}$ This yields another application of the results of [29] since only the case $A\left(d_{j}\right)$ differential was considered in the examples given in that paper.
} 
defines a global density on $M$ which integrates to the extended Wodzicki residue

$$
\operatorname{Res}(A \log Q):=\int_{M} \operatorname{res}_{x}(A \log Q) d x
$$

of $A \log Q$.

The zeta function $\zeta(A, Q)$ is holomorphic at zero and we have

$$
\zeta(A, Q)(0)=\lim _{z \rightarrow 0} \zeta(A, Q)(z)=\operatorname{Tr}(T)-\frac{1}{q} \operatorname{Res}(A \log Q)
$$

When $A$ is a differential operator, its $Q$-weighted $\zeta$-regularised trace $\zeta(A, Q)(0)$ is therefore proportional to the extended Wodzicki residue $\operatorname{Res}(A \log Q)$. So in that case, the $\zeta$-regularised trace $\zeta(A, Q)(0)$ is local since the extended Wodzicki residue is local in so far as it is expressed as an integral over $M$ of the pointwise extended residue (Proposition 1.8), which only depends on finitely many (here one) homogeneous components of the symbol of $A$.

In particular, for $A=I$, we have as announced previously, that the logarithmic residue $\operatorname{Res}(\log Q)$ is well defined, moreover the zeta function of $Q$ at zero is local

$$
\zeta_{Q}(0):=\zeta(I, Q)(0)=-\frac{1}{q} \operatorname{Res}(\log Q) .
$$

\section{Heat-kernel expansions revisited}

\subsection{The heat-kernel expansion in terms of Wodzicki residues}

We recall the definition and some properties of the Mellin transform following [9] (see also [18]).

Definition 2.1. Let $f(t)$ be a locally Lebesgue integrable function over $] 0,+\infty[$. The Mellin transform of $f(t)$ is defined as

$$
\mathcal{M}(f)(z):=\int_{0}^{\infty} f(t) t^{z-1} d t
$$

The largest open strip $a<\Re(z)<b$ in which the integral converges is called the fundamental strip.

We recall the following well-known Inverse Mellin Mapping Theorem [9, Theorem 4].

Proposition 2.2. Let $f(t)$ be continuous in $] 0,+\infty[$ with Mellin transform $\phi(z)$ having a fundamental strip $a<\Re(z)<b$.

1. Provided

(a) $\phi(z)$ admits a meromorphic continuation to the strip $(\gamma, b)$ for some $\gamma<a$ with a finite number of poles in the strip, and is analytic on $\Re(z)=\gamma$,

(b) there exists a real number $c$ in $(a, b)$ such that for some $r>1$

$$
\phi(z)=O\left(|z|^{-r}\right), \text { when }|z| \rightarrow \infty \text { in } \gamma \leq \Re(z) \leq c \text {, }
$$


(c) $\phi$ admits the singular expansion for $z \in(\gamma, a)$

$$
\phi(z) \cong \sum_{i \geq 0} \sum_{j \geq 0} a_{i, j} \frac{(-1)^{k_{j}} k_{j} !}{\left(z-d_{j}\right)^{k_{i}+1}},
$$

then $f$ admits an asymptotic expansion at 0 given by

$$
f(t)=\sum_{i \geq 0} \sum_{j \geq 0} a_{i, j} t^{-d_{j}} \log ^{k_{i}} t+O\left(t^{-\gamma}\right) .
$$

2. Provided

(a) $\phi(z)$ admits a meromorphic continuation to the strip $(a, \gamma)$ for some $\gamma>b$ with a finite number of poles in the strip, and is analytic on $\Re(z)=\gamma$,

(b) there exists a real number $c$ in $(a, b)$ such that for some $r>1$

$$
\phi(z)=O\left(|z|^{-r}\right) \text {, when }|z| \rightarrow \infty \text { in } c \leq \Re(z) \leq \gamma,
$$

(c) $\phi$ admits the singular expansion for $z \in(c, \gamma)$,

$$
\phi(z) \cong \sum_{i \geq 0} \sum_{j \geq 0} a_{i, j} \frac{(-1)^{k_{i}} k_{i} !}{\left(z-d_{j}\right)^{k_{i}+1}},
$$

then $f$ admits an asymptotic expansion at $\infty$ given by

$$
f(t)=\sum_{i \geq 0} \sum_{j \geq 0} a_{i, j} t^{-d_{j}} \log ^{k_{i}} t+O\left(t^{-\gamma}\right) .
$$

Remark 2.3. In particular, it follows from 2.c) that if $\phi$ is analytic in some half plane $\Re(z)>c$ then $f(t)=O\left(t^{-\gamma}\right)$ for any $\gamma>c$ and $f$ is a Schwartz function.

Here is a useful example to keep in mind for what follows.

Example 2.4. For any $\lambda>0$ the map $z \longmapsto \phi(z)=\Gamma(z) \lambda^{-z}$ satisfies the above assumptions as a result of the properties of the Gamma function. Indeed, on the one hand it is meromorphic on the complex plane with simple poles in $\mathbb{Z}_{\leq 0}$. On the other hand, it follows from Stirling's formula (see e.g. 10, Proposition IV:1.14]) which expresses the Gamma function as $\Gamma(z)=\sqrt{2 \pi} z^{z-\frac{1}{2}} e^{-z} e^{H(z)}$ for some function $H$ given by a series, that for any $0<\gamma<\delta$ there is a positive constant $C_{\gamma, \delta}$ such that for any

$$
\gamma \leq \Re(z) \leq \delta \Longrightarrow|\Gamma(z)| \leq C_{\gamma, \delta}|\Im(z)|^{\delta-\frac{1}{2}} e^{-\gamma} .
$$

Since $\lambda^{-z}$ is bounded from above by $\lambda^{-\gamma}$ this shows that the Condition 1. b) is satisfied for any $r>1$. The inverse Mellin transform is $f(t)=e^{-t \lambda}$ which defines a Schwartz function on $\mathbb{R}_{+}$.

Combining these properties of the Gamma function with the results of Theorem 1.15 yields the following useful properties.

Corollary 2.5. Let $A(z) \in \Psi(M, E)$ be a holomorphic family of affine order $\alpha(z)=a-q z$ for some positive real number $q$; we set

$$
\phi(z):=\Gamma(z) \operatorname{TR}(A(z)) \quad \alpha:=\operatorname{Max}\left\{0 ; \frac{a+n}{q}\right\} .
$$


1. The map $\phi$ is holomorphic on the half-plane $\Re(z)>\alpha$, it is analytic on any imaginary line $\Re(z)=\gamma$ for $\gamma>\alpha$, and $(\alpha, \infty)$ is the largest open strip where $\phi$ is defined.

2. $\phi$ admits a meromorphic extension to the whole complex plane with countably many simple poles $\left\{d_{j}:=\frac{a+n-j}{q}, j \in \mathbb{Z}_{\geq 0}\right\} \cup \mathbb{Z}_{\leq 0}$

3. whenever $z \mapsto \operatorname{TR}(A(z))$ is uniformly bounded in closed strips $\frac{a+n}{q}<\gamma \leq$ $\Re(z) \leq \delta$, then for some $r>1$ we have the following asymptotic behaviour of $\phi$ at infinity along imaginary lines

$$
\phi(z)=O\left(|z|^{-r}\right), \text { when }|z| \rightarrow \infty \text { in } \frac{a+n}{q}<\gamma \leq \Re(z) \leq \delta,
$$

as a consequence of the corresponding property of the Gamma function described above.

4. $\phi$ admits a singular expansion on any horizontally bounded strip

$$
\phi(z) \cong \sum_{j=0} \frac{a_{j}}{z-d_{j}},
$$

as a consequence of the second item.

Let as before, $M$ be an $n$-dimensional closed manifold and $E$ be a finite rank vector bundle over $M$. The subsequent theorem follows from Proposition 2.2 applied to $\phi(z)=\Gamma(z) \operatorname{TR}(A(z))$.

Theorem 2.6. Let $\mathcal{A}: z \mapsto A(z) \in \Psi(M, E)$ be some holomorphic family of operators of affine order $\alpha(z)=a-q z$ with $q$ some positive real number corresponding to the Mellin transform of some analytic family $\widetilde{A}(t), t>0$ of trace-class operators in $\Psi(M, E)$. Whenever $z \mapsto \mathrm{TR}(A(z))$ is uniformly bounded on closed strips $\frac{a+n}{q}<\gamma \leq \Re(z) \leq \delta$, then $f_{\mathcal{A}}(t)=\operatorname{Tr}(\widetilde{A}(t))$ admits an asymptotic expansion at 0 given by

$$
f_{\mathcal{A}}(t) \sim_{0} \frac{1}{q} \sum_{j \geq 0} a_{j} t^{\frac{-a-n+j}{q}}
$$

with

$$
a_{j}=-\frac{1}{q} \operatorname{Res}\left(A\left(d_{j}\right)\right) \quad \text { for } \quad j>a+n .
$$

If $A(0)$ differs from a differential operator by a trace-class pseudodifferential operator $T$, the constant term in the expansion (29) which coincides with the constant term in the Laurent expansion of $\mathrm{TR}(A(z))$ reads

$$
a_{j}=\operatorname{Tr}(T)-\frac{1}{q} \operatorname{Res}\left(A^{\prime}(0)\right), \quad \text { for } \quad j=a+n .
$$

Let $Q \in \Psi(M, E)$ be a weight of positive order $q$. We further assume that it is "close" to a positive operator i.e., its spectrum is concentrated in a cone centered around the positive real line; in particular, it has spectral cut $\beta=\pi$.

The holomorphic family $\mathcal{A}: z \mapsto A(z)=A Q^{-z}$ is the Mellin transform of the analytic family $A e^{-t Q}, t>0$. Since the map $z \mapsto \operatorname{TR}(A(z))$ is uniformly bounded on closed strips $\frac{a+n}{q}<\gamma \leq \Re(z) \leq \delta$ we can apply Theorem 2.6, which yields the following corollary. 
Corollary 2.7. The inverse Mellin transform

$$
f_{\mathcal{A}}(t):=\operatorname{Tr}\left(A e^{-t Q}\right)
$$

of $\phi_{\mathcal{A}}(z):=\Gamma(z) \mathrm{TR}\left(A Q^{-z}\right)$ is a Schwartz function on $] 0,+\infty[$, which admits an asymptotic expansion at 0 given by

$$
f_{\mathcal{A}}(t) \sim_{0} t^{-\frac{a+n}{q}} \sum_{j \geq 0} a_{j} t^{\frac{j}{q}}
$$

where

$$
\begin{aligned}
a_{j} & =-\frac{1}{q} \operatorname{Res}\left(A Q^{\frac{-a-n+j}{q}}\right) \quad \text { for } \quad j<a+n \\
\text { and } a_{a+n} & =\zeta(A, Q)(0)=\operatorname{Tr}(T)-\frac{1}{q} \operatorname{Res}(A \log Q)
\end{aligned}
$$

if $A \in \Psi(M, E)$ differs from a differential operator by a trace-class operator $T$.

Let $\Delta \in \Theta(M, E)$ be an admissible operator and let $E$ be equipped with a hermitian metric, which combined with a Riemannian metric on $M$ induces a (weak) inner product on $C^{\infty}(M, E)$. We previously saw that the operator $Q:=$ $\Delta+\pi_{\Delta}$ defines a weight.

Notation convention: Integrating over $M$ the pointwise residue in (16) gives rise to the logarithmic residue of $\Delta$ defined as

$$
\operatorname{Res}(\log \Delta):=\operatorname{Res}\left(\log \left(\Delta+\pi_{\Delta}\right)\right) .
$$

Similarly, for any $x \in M$ and $\alpha \in \mathbb{R}$ we set

$$
\operatorname{res}_{x}\left(\Delta^{\alpha}\right):=\operatorname{res}_{x}\left(\left(\Delta+\pi_{\Delta}\right)^{\alpha}\right) ; \quad \operatorname{Res}\left(\phi \Delta^{\alpha}\right):=\operatorname{Res}\left(\phi\left(\Delta+\pi_{\Delta}\right)^{\alpha}\right) .
$$

We now specialise to the multiplication operator $A=\phi \in C^{\infty}(M)$ and apply Theorem 2.6 to the holomorphic family $A(z)=\phi\left(\Delta+\pi_{\Delta}\right)^{-z}$.

Theorem 2.8. For any smooth function $\phi \in C^{\infty}(M)$ we have

$$
\begin{aligned}
& \operatorname{Tr}\left(\phi e^{-t \Delta}\right) \sim_{t \rightarrow 0} \\
& \quad-\frac{(4 \pi)^{\frac{n}{2}}}{2}\left[\operatorname{Res}(\phi \log \Delta) \delta_{\frac{n}{2}-\left[\frac{n}{2}\right]}\right. \\
& \left.\quad+\sum_{k \in\left[0, \frac{n}{2}[\cap \mathbb{Z}\right.} \Gamma\left(\frac{n}{2}-k\right) \operatorname{Res}\left(\phi \Delta^{k-\frac{n}{2}}\right) t^{k-\frac{n}{2}}\right] .
\end{aligned}
$$

The local heat-kernel trace $K_{t}(\Delta)(x, x)$ of the operator $\Delta$ at the point $x$ is defined by

$$
\operatorname{Tr}\left(\phi e^{-t \Delta}\right)=\int_{M} \phi(x) K_{t}(\Delta)(x, x) \sqrt{\operatorname{det} g}(x) d x \quad \forall \phi \in C^{\infty}(M)
$$

and therefore it has the following asymptotic expansion

$$
\begin{aligned}
& K_{t}(\Delta)(x, x) \sim_{t \rightarrow 0} \\
& -\frac{(4 \pi)^{\frac{n}{2}}}{2 \sqrt{\operatorname{det} g}(x)}\left[\operatorname{res}_{x}(\log \Delta) \delta_{\frac{n}{2}-\left[\frac{n}{2}\right]}\right. \\
& \left.+\sum_{k \in\left[0, \frac{n}{2}[\cap \mathbb{Z}\right.} \Gamma\left(\frac{n}{2}-k\right) \operatorname{res}_{x}\left(\Delta^{k-\frac{n}{2}}\right) t^{k-\frac{n}{2}}\right] .
\end{aligned}
$$


Remark 2.9. Formula (35) compares with known formulae for the spectral action (take $f(\lambda)=e^{-t \lambda}$ in [5. Formula 1.5]), in which the non-constant coefficients arise as Dixmier traces.

Proof. It follows from (28) that the constant term in the heat-kernel expansion reads

$$
\mathrm{fp}_{t=0} \operatorname{Tr}\left(\phi e^{-t\left(\Delta+\pi_{\Delta}\right)}\right)=\zeta\left(\phi, \Delta+\pi_{\Delta}\right)(0)=\frac{1}{2} \operatorname{Res}(\phi \log \Delta) .
$$

Formula (35) then follows from Corollary 2.7 with $q=2$ (cf. footnote in the introduction).

Since this holds for any smooth function $\phi$, formula (36) follows.

Remark 2.10. Combining (34) with (16) applied to $\Delta$

$$
\operatorname{res}_{x}(\log \Delta)=\frac{1}{2 \pi i} \int_{|\xi|_{x}=1} \int_{\Gamma} \log \lambda \sigma_{-q-n}\left(\Delta+\pi_{\Delta}-\lambda\right)^{-1}(x, \xi) d \lambda d_{S} \xi
$$

yields

$$
a_{\frac{n}{2}}(x)=-\frac{1}{4 i \pi^{\frac{n}{2}+1} \sqrt{\operatorname{det} g}(x)} \int_{|\xi|_{x}=1} \int_{\Gamma} \log \lambda \sigma_{-q-n}\left(\Delta+\pi_{\Delta}-\lambda\right)^{-1}(x, \xi) d \lambda d_{S} \xi
$$

This compares with similar formulae in the literature as for example [3, (2.11)]

$$
a_{\frac{n}{2}}(x)=\frac{1}{2} \int_{|\xi|_{x}=1} \int_{0}^{\infty} \sigma_{-q-n}\left(\Delta+\pi_{\Delta}-\lambda\right)^{-1}(x, \xi) d \lambda d_{S} \xi .
$$

\subsection{The case of geometric operators}

We now single out a class of differential operators we call geometric differential operators (also considered in [25]) i.e., differential operators $A=\sum_{|\alpha| \leq d} a_{\alpha}(x) D_{x}^{\alpha} \in$ $\Theta(M, E)$ where $d$ is the order of the operator and whose coefficients $a_{\alpha}(x)$ are given by an algebraic expression in terms of the jets at the point $x$ of the metric on $M$ and a connection on $E$.

The Laplace-Beltrami operator $\Delta_{g}$ on a closed Riemannian manifold $(M, g)$ is a geometric differential operator of order 2. Another example is the BochnerLaplacian

$$
\Delta^{\nabla}:=\operatorname{Tr}\left(\nabla^{T^{*} M \otimes E} \circ \nabla^{E}\right) \in \Psi(M, E)
$$

built from a connection $\nabla^{E}$ on $E$ and the induced connection $\nabla^{T^{*} M \otimes E}$ on the tensor product $T^{*} M \otimes E$. Here the trace is taken over the two factors of $T^{*} M$. The square of a Dirac operator on a spin manifold $(M, g)$, which differs from the corresponding Bochner-Laplacian by a term proportional to the scalar curvature, is geometric. More generally, the square $A=D^{2}$ of a Dirac-type operator $D=\sum_{i=1}^{n} c\left(e_{i}\right) \nabla_{i}^{E}$ is a geometric operator; here $\nabla^{E}$ is a Clifford connection on a Clifford bundle $E$ over $M, c$ the Clifford multiplication and $e_{i}, i=1, \cdots, n$ an orthonormal frame of the cotangent bundle at a point $x$. All these examples fall in the class of Laplace-type differential operators considered previously.

Proposition 2.11. Let $A$ and $\Delta$ be two geometric differential operators in $\Theta(M, E)$ and let $\Delta$ be admissible. The extended local residue $\operatorname{res}_{x}(A \log \Delta)$ at a point $x$ and for any real number $\alpha$, the local residue $\operatorname{res}_{x}\left(A \Delta^{\alpha}\right)$, are algebraic expressions of the jets of the metric and the connection. 
Proof. That $\operatorname{res}_{x}(A \log \Delta)$ is an algebraic expression in the $x$-jets of a finite number of homogeneous components of the symbols follows from Proposition 1.8, which tells us that the extended local residue is an algebraic expression in the $x$-jets of a finite number of homogeneous components of the symbols of $A$ and $\Delta$ combined with the fact that these homogeneous components are themselves algebraic expressions in the jets of the metric and the connection.

That $\operatorname{res}_{x}\left(A \Delta^{\alpha}\right)$ is an algebraic expression in the $x$-jets of a finite number of homogeneous components of the symbols can be shown similarly.

The case of the Laplace-Beltrami operator $\Delta_{g}$ on an $n$-dimensional Riemannian manifold $(M, g)$ is particularly relevant for us since it enables to capture the scalar curvature as a Wodzicki (possibly extended, depending on the dimension) residue. Indeed, the scalar curvature $\mathfrak{s}_{g}$ is proportional to the coefficient $b_{1}(x)$ in the heatexpansion

$$
\begin{aligned}
K_{t}\left(\Delta_{g}\right)(x, x) \quad \sim_{t \rightarrow 0} \quad t^{-n \frac{n}{2}} \sum_{j \geq 0} a_{k}(x) t^{\frac{k}{2}} \\
=t^{-\frac{n}{2}} \sum_{j \geq 0} b_{j}(x) t^{j}
\end{aligned}
$$

where we have set $b_{j}=a_{2 j}$ since the coefficients $a_{2 j+1}$ vanish [15], 31].

We have [26, Formula (5a)]

$$
\mathfrak{s}_{g}=3 b_{1},
$$

independently of the dimension $n$.

This combined with Theorem 2.8 leads to the following expressions of the scalar curvature in terms of Wodzicki residues.

Proposition 2.12. When $n=2$ we have

$$
\mathfrak{s}_{g}(x)=-\frac{6 \pi}{\sqrt{\operatorname{det} g}(x)} \operatorname{res}_{x}(\log \Delta) .
$$

If $n>2$ then,

$$
\mathfrak{s}_{g}(x)=-\frac{3}{2}(4 \pi)^{\frac{n}{2}} \Gamma\left(\frac{n}{2}-1\right) \frac{\operatorname{res}_{x}\left(\left(\Delta+\pi_{\Delta}\right)^{1-\frac{n}{2}}\right)}{\sqrt{\operatorname{det} g}(x)} .
$$

In dimension 2 , which is the case we are going to focus on in the sequel, we have

$$
\left\langle\mathfrak{s}_{g}, \phi\right\rangle_{g}=-6 \pi \operatorname{Res}\left(\phi \log \Delta_{g}\right) \quad \forall \phi \in C^{\infty}(M)
$$

\section{Holomorphic families on Hilbert modules}

We carry out to pseudodifferential operators on bundles of von Neumann Hilbert modules the constructions of Section 1 on closed manifolds.

\subsection{Finite type Hilbert modules}

We start out recalling the setup of finite type Hilbert modules closely following 3 ] and 349

\footnotetext{
${ }^{9}$ Note that in Schick's paper these objects are called $\mathcal{A}$-Hilbert spaces to distinguish them
} from Hilbert $C^{*}$-modules. 
Let $\mathcal{A}$ be a von Neumann algebra equipped with a finite trace $\tau: \mathcal{A} \rightarrow \mathbb{C}$. This means that $\mathcal{A}$ is a unital $\mathbb{C}$-algebra with a $\star$ operation, and the following properties are satisfied:

1. $(\cdot, \cdot): \mathcal{A} \times \mathcal{A} \longrightarrow \mathbb{C}$, defined by $(a, b):=\tau\left(a b^{\star}\right)$ is a scalar product and the completion $\mathcal{A}_{2}$ with respect to this scalar product is a separable Hilbert space.

2. $\mathcal{A}$ is weakly closed when viewed as a subalgebra of the space $\mathcal{L}\left(\mathcal{A}_{2}\right)$ of linear, bounded operators on $\mathcal{A}_{2}$ (identifying elements of $\mathcal{A}$ with the corresponding left translations in $\left.\mathcal{L}\left(\mathcal{A}_{2}\right)\right)$.

3. The trace is normal, i.e. for any monotone increasing net, $\left(a_{i}\right)_{i \in I}$ such that $a_{i} \geq 0$ and $a=\sup _{i \in I} a_{i}$ exists in $\mathcal{A}$, one has $\operatorname{tr}_{\tau}(a)=\sup _{i \in I} \operatorname{tr}_{\tau}\left(a_{i}\right)$.

A right $\mathcal{A}$-Hilbert module is a Hilbert space $\mathcal{W}$ with a continuous right $\mathcal{A}$-action that admits an $\mathcal{A}$-linear isometric embedding into $\mathcal{A}_{2} \otimes H$ for some Hilbert space $H$. The Hilbert module $\mathcal{W}$ is called of finite type if the space $H$ can be chosen finite dimensional. We denote by $\mathcal{L}_{\mathcal{A}}(\mathcal{W})$ the von Neumann algebra of bounded $\mathcal{A}$-linear operators on $\mathcal{W}$. The (unbounded) trace on $\mathcal{L}_{\mathcal{A}}(\mathcal{W})$ induced by $\tau$ and by the usual trace on $\mathcal{L}(H)$ is denoted $\operatorname{tr}_{\tau}$.

Example 3.1. Let $\Gamma$ be a countable group and $\ell^{2}(\Gamma)$ be the Hilbert space of square integrable complex valued functions on $\Gamma$. The von Neumann algebra $\mathcal{N} \Gamma$ consists by definition of all bounded operators on $\ell^{2}(\Gamma)$ that commute with the left convolution action of $\Gamma$. It contains $\mathbb{C} \Gamma$ as a weakly dense subset, and on $\mathbb{C} \Gamma$ the canonical trace $\tau$ is given by

$$
\tau\left(\sum a_{\gamma} \gamma\right)=a_{e}
$$

where $e$ is the unit element in $\Gamma$. Then $\mathcal{W}=\ell^{2}(\Gamma)$ is a finite type $\mathcal{N} \Gamma$-module, indeed $\ell^{2}(\Gamma) \simeq(\mathcal{N} \Gamma)_{2}$.

Example 3.2. In particular, for $\Gamma=\mathbb{Z}^{n}$, the Fourier transform gives an isometric $\mathbb{Z}^{n}$-equivariant isomorphism $\ell^{2}\left(\mathbb{Z}^{n}\right) \rightarrow L^{2}\left(\mathbb{T}^{n}\right)$, where $\mathbb{T}^{n}$ is the $n$-dimensional torus. Therefore $\mathcal{N} \mathbb{Z}^{n}$ coincides with the commutant $\mathcal{L}\left(L^{2}\left(T^{n}\right)\right)^{\mathbb{Z}_{n}}$ of the $\mathbb{Z}_{n}$-action on $L^{2}\left(\mathbb{T}^{n}\right)$, and one obtains an isomorphism $\mathcal{N} \mathbb{Z}^{n} \simeq L^{\infty}\left(\mathbb{T}^{n}\right)$. The canonical trace $\tau: L^{\infty}\left(\mathbb{T}^{n}\right) \rightarrow \mathbb{C}$ is

$$
\tau(f)=\int_{\mathbb{T}^{n}} f d \mu
$$

where $\mu$ is the measure on $\mathbb{T}^{n}$ induced by the canonical Lebesgue measure on $\mathbb{R}^{n}$.

On a manifold $M$, an $\mathcal{A}$-Hilbert module bundle $\mathcal{E} \rightarrow M$ is a locally trivial bundle with fibre a (finitely generated, projective) $\mathcal{A}$-Hilbert module $\mathcal{W}$, the transition functions being isometries of $\mathcal{A}$-Hilbert modules.

Remark 3.3. The space of $L^{2}$-sections of an $\mathcal{A}$-Hilbert module bundle $\mathcal{E} \rightarrow M$ is an $\mathcal{A}$-Hilbert module. In fact, if $U$ is a subset of $M$ such that $M \backslash U$ has measure zero and $\mathcal{E}_{\mid U} \simeq U \times \mathcal{W}$ (where $\mathcal{W}$ is the fibre), then $L^{2}(M, \mathcal{E}) \simeq L^{2}\left(U, \mathcal{E}_{\mid U}\right) \simeq$ $L^{2}(U) \otimes \mathcal{W}$. The set $U$ can be chosen for example as being the union of the interiors of the top-order cells of a triangulation of $M$.

Example 3.4. (Flat $\mathcal{N} \Gamma$-Hilbert module) Let $M$ be a closed manifold with $\pi_{1}(M)=$ $\Gamma$, let $\pi: \widetilde{M} \rightarrow M$ be a universal covering of $M$, with $\Gamma$ acting on the right by 
deck transformations. Because the left $\Gamma$-action and the right $\mathcal{N} \Gamma$-action on $\ell^{2}(\Gamma)$ commute, $\mathcal{H}:=\widetilde{M} \times_{\Gamma} \ell^{2}(\Gamma)$ is a finitely generated projective bundle of (right) $\mathcal{N} \Gamma$-Hilbert modules over $M$. Moreover, $\mathcal{H}$ is endowed with a flat structure since the transition functions are locally constant.

The flat bundle $\mathcal{H} \rightarrow M$ can be used to describe the analysis on the universal covering. In fact, there is a well-known correspondence between $L^{2}(M, \mathcal{H})$ and $L^{2}(\widetilde{M})$, which translates twisted differential operators on one hand with $\Gamma$ invariant differential operators on the other. We refer for example to [34, 7.5] or [30. Prop. E.6] for the complete dictionary for spaces, operators, and $L^{2}$-invariants. Let us illustrate this in the case of the $n$-torus discussed above.

Example 3.5. To the universal covering $\pi: \mathbb{R}^{n} \rightarrow \mathbb{T}^{n}$ with fundamental group $\mathbb{Z}^{n}$ corresponds the finitely generated projective bundle $\mathcal{H}:=\mathbb{R}^{n} \times_{\mathbb{Z}^{n}} \ell^{2}\left(\mathbb{Z}^{n}\right)$ of (right) $L^{\infty}\left(\mathbb{T}^{n}\right)$-Hilbert modules over $\mathbb{T}^{n}$. In this correspondence $L^{2}$-functions on $\mathbb{R}^{n}$ are viewed as $L^{2}$-sections of the bundle $\mathcal{H}$ over $\mathbb{T}^{n}$ via the map which sends $f$ to $\hat{f}: x \mapsto \sum_{\gamma \in \mathbb{Z}^{n}} f\left(\gamma \pi^{-1}(x)\right) \otimes\left[\pi^{-1}(x), \gamma\right]$. This induces an isometry

$$
\Phi: L^{2}\left(\mathbb{R}^{n}\right) \simeq L^{2}\left(\mathbb{T}^{n}\right) \otimes \ell^{2}\left(\mathbb{Z}^{n}\right) \longrightarrow L^{2}\left(\mathbb{T}^{n}, \mathcal{H}\right)
$$

which sends $\left\{f \in C^{\infty}\left(\mathbb{R}^{n}\right): \sum_{\gamma \in \mathbb{Z}^{n}}|f(\gamma x)|^{2}<\infty \forall x \in \mathbb{R}^{n}\right\}$ to $C^{\infty}\left(\mathbb{T}^{n}, \mathcal{H}\right)$.

\subsection{Pseudodifferential operators on bundles of Hilbert mod- ules}

We describe pseudodifferential operators on bundles of Hilbert modules, following [3, 2.2-4].

Let $(M, g)$ be a closed Riemannian manifold of dimension $n$ and let $p: \mathcal{E} \rightarrow M$ be a bundle of finitely generated projective $\mathcal{A}$-Hilbert modules with fibre $\mathcal{W}$.

A linear operator $A: C^{\infty}(M, \mathcal{E}) \rightarrow C^{\infty}(M, \mathcal{E})$ is a (classical) pseudodifferential $\mathcal{A}$ operator of order $a \in \mathbb{C}$ if in some atlas of $\mathcal{E} \rightarrow M$ it is of the form $A=\sum_{j=1}^{J} A_{j}+R$ where

- $R: C^{\infty}(M, \mathcal{E}) \rightarrow C^{\infty}(M, \mathcal{E})$ is a smoothing operator, namely with Schwartz kernel given by a smooth section of the bundle $\mathcal{L}_{\mathcal{A}} \rightarrow M \times M$ of $\mathcal{A}$-linear bounded operators whose fibre at $(x, y) \in M \times M$ is the Banach space of $\mathcal{A}$-linear operators from the fibre $\mathcal{E}_{y}$ to the fibre $\mathcal{E}_{x}$,

- the operators $A_{j}$ are properly supported operators (meaning that the canonical projections $M \times M \rightarrow M$ restricted to the support of the Schwartz kernel are proper maps) from $C^{\infty}(M, \mathcal{E})$ into itself. This implies in particular that the $A_{j}$ send $C_{0}^{\infty}(M, \mathcal{E})$ into itself. In any coordinate chart $A_{j}$ is therefore of the form given in (7), for some symbol $\sigma \in C^{\infty}\left(U \times \mathbb{R}^{n}, \operatorname{End}_{\mathcal{A}} \mathcal{E}\right)$ which is asymptotically polyhomogeneous at infinity.

For $a \in \mathbb{C}$, let $\Psi^{a}(M, \mathcal{E})$ be the set of classical pseudodifferential operators of order $a$; then $\Psi^{-\infty}(M, \mathcal{E}):=\cap_{a} \Psi^{a}(M, \mathcal{E})$ corresponds to the smoothing operators, and $\Psi(M, \mathcal{E}):=\cup_{a} \Psi^{a}(M, \mathcal{E})$. The subalgebra of differential operators will be denoted $\Theta(M, \mathcal{E})$.

Remark 3.6. Going back to the example of the $n$-torus $\mathbb{T}^{n}$ and its $\mathbb{Z}^{n}$-covering $\pi: \mathbb{R}^{n} \rightarrow \mathbb{T}^{n}$, we know that $\mathbb{Z}^{n}$-invariant (i.e., $A \circ t_{a}^{*}=t_{a}^{*} \circ A$ for any $a \in \mathbb{Z}^{n}$ with $\left.t_{a}(x)=x+a\right)$ differential operators on $\mathbb{R}^{n}$ give rise to elements of $\Theta(M, \mathcal{H})$. This 
raises the question how the algebra $\Psi\left(\mathbb{T}^{n}, \mathcal{H}\right)$ relates to the algebra of $\mathbb{Z}^{n}$-invariant classical pseudodifferential operators on $\mathbb{R}^{n}$ studied by Ruzhanski and Turunen in [33] or equivalently the $\theta=0$ instance of the algebra $\Psi\left(\mathbb{T}_{\theta}^{n}\right)$ described in Section 4.

For any $A \in \Psi^{a}(M, \mathcal{E}), a \in \mathbb{R}, A$ defines a bounded linear map on the $H^{s_{\text {- }}}$ Sobolev closure $H^{s}(M, \mathcal{E})$ of $C^{\infty}(M, \mathcal{E})$ with values in $H^{s-a}(M, \mathcal{E})$, for any $s \in \mathbb{R}$. We refer the reader to [3, §2.2] for Sobolev closures and to [3, Prop. 2.7] for the property in the case $s=a$.

The notions of admissibility (Definition 1.3) and Agmon angle (Lemma 1.6) carry out from the closed manifold case to the setup of pseudodifferential operators on bundles of von Neumann Hilbert modules, taking into account that here the spectrum is not necessarily purely discrete.

Definition 3.7. Let $A$ be an operator in $\Psi^{a}(M, \mathcal{E})$. For an angle $\beta$ and for $\epsilon>0$, denote $V_{\beta, \epsilon}:=\{z \in \mathbb{C}:|z|<\epsilon\} \cup\{z \in \mathbb{C} \backslash 0: \arg z \in(\beta-\epsilon, \beta+\epsilon)\}$. Then $\beta$ is called an Agmon angle for $A$ if there is some $\epsilon>0$ such that $\operatorname{sp}(A) \cap V_{\beta, \epsilon}=\emptyset$, where $\operatorname{sp}(A)$ stands for the spectrum of $A$.

In particular an operator with Agmon angle is elliptic and invertible.

Definition 3.8. We call weight an operator in $\Psi(M, \mathcal{E})$ of positive order that admits an Agmon angle. We call admissible an operator $A \in \Psi(M, \mathcal{E})$ which is a weight modulo a smoothing perturbation, i.e. if it has positive order, and there exists a smoothing operator $R$ such that $A+R$ has an Agmon angle.

The constructions of complex powers and the logarithm recalled in Section 1.2 extend word for word to the setting of Hilbert modules.

Let $A$ be a weight in $\Psi^{a}(M, \mathcal{E})$ with spectral cut $\beta$. Then for $\Re(z)>0$, its complex powers (see [3, (2.8)] for further details, and compare with (12))

$$
A_{\beta}^{z}=\frac{i}{2 \pi} \int_{\Gamma_{\beta}} \lambda^{z}(\lambda-A)^{-1} d \lambda,
$$

and respectively the operators (as in (13))

$$
L_{\beta}(A, z)=\frac{i}{2 \pi} \int_{\Gamma_{\beta}} \log _{\beta} \lambda \lambda^{z}(\lambda-A)^{-1} d \lambda,
$$

are well defined bounded linear maps from $H^{s}(M, \mathcal{E}), s \in \mathbb{R}$, with values in $H^{s-a \Re(z)}(M, \mathcal{E})$ respectively in $H^{s-a \Re(z)+\epsilon}(M, \mathcal{E})$, for any $\epsilon>0$. Here $\Gamma_{\beta}$ is a closed contour in $\mathbb{C} \backslash\left\{r e^{i \beta}, r \geq 0\right\}$ around the spectrum of $A$ oriented clockwise. These definitions extend to the whole plane inductively on $k \in \mathbb{N}$ setting

$$
A_{\beta}^{z}:=A^{k} A_{\beta}^{z-k} \text {, respectively } L_{\beta}(A, z):=A^{k} L_{\beta}(A, z-k) \text {, for } \Re(z)<k \text {. }
$$

$A_{\beta}^{z}$ is an operator in $\Psi(M, \mathcal{E})$ of order $a z$ for any complex number $z$, and the logarithm

$$
\log _{\beta}(A):=L_{\beta}(A, 0)
$$

of $A$ is a bounded linear map from $H^{s}(M, \mathcal{E})$ to $H^{s+\epsilon}(M, \mathcal{E}), \forall \epsilon>0$. One has by construction $\log _{\beta} A A_{\beta}^{z}=A_{\beta}^{z} \log _{\beta} A, \forall z \in \mathbb{C}$.

Remark 3.9. Using the same convention as in Remark 1.7. we usually drop the explicit mention of the dependence of $\beta$. 
In a local trivialisation, the symbol of $\log _{\beta} A$ reads (see e.g. [36])

$$
\sigma\left(\log _{\beta} A\right)(x, \xi)=a \log |\xi| I+\sigma_{\mathrm{cl}}\left(\log _{\beta} A\right)(x, \xi)
$$

where $a$ denotes the order of $A$ and $\sigma_{\mathrm{cl}}(\log A)$ is a classical symbol of order zero with homogeneous components $\sigma_{-j}(\log A)$ of degree $-j, j \in \mathbb{Z}_{\geq 0}$.

\subsection{The extended $\tau$-Wodzicki residue}

Mimicking the definition of the pointwise residue (10) on closed manifolds, for any $A \in \Psi(M, \mathcal{E})$ with symbol $\sigma(A)$ in a local chart of $M$ at a point $x$, we call

$$
\operatorname{res}_{x}^{\tau}(A):=\int_{|\xi|_{x}=1} \operatorname{tr}^{\tau}\left(\sigma_{-n}(A)\right)(x, \xi) d_{S} \xi,
$$

the pointwise $\tau$-Wodzicki residue of $A$. As in the closed manifold case, one proves that $\operatorname{res}_{x}^{\tau}(\sigma(A)) d x$ defines a global density on $M$ [39], so we can define the $\tau$ Wodzicki residue of $A$ (compare with (11))

$$
\operatorname{Res}^{\tau}(A)=\int_{M} \operatorname{res}_{x}^{\tau}(A) d x=\int_{M} d x \int_{|\xi|_{x}=1} \operatorname{tr}^{\tau}\left(\sigma_{-n}(A)(x, \xi)\right) d_{S} \xi
$$

Remark 3.10. Definition (46) relates to the definitions of the Wodzicki residue by Benameur-Fack 2, Def. 9] and Vassout [38 in the context of measured foliations.

The pointwise Wodzicki $\tau$-residue extends to the $\operatorname{logarithm} \log A$ of an admissible invertible operator $A \in \Psi(M, \mathcal{E})$. At a point $x$ we set

$$
\operatorname{res}_{x}^{\tau}(\log A):=\int_{|\xi|_{x}=1} \operatorname{tr}^{\tau}\left(\sigma_{-n}(\log A)\right)(x, \xi) d_{S} \xi,
$$

Mimicking the definition (18), for any admissible operator $A \in \Psi(M, \mathcal{E})$, we set

$$
\operatorname{res}_{x}^{\tau}(\log A):=\operatorname{res}_{x}^{\tau}(\log (A+R)) \quad \forall x \in M,
$$

where $R$ is any smoothing operator such that $A+R$ has an Agmon angle.

In the subsequent paragraph, we show as in the closed manifold case, that $\operatorname{res}_{x}^{\tau}(\log A) d x$ defines a global density.

\subsection{The $\tau$-Wodzicki residue as a complex residue}

For holomorphic families of operators in $\Psi(M, \mathcal{E})$, whose explicit definition we omit here since they are defined in the same manner as in the closed case (see Definition [1.14), we give the Hilbert-module counterpart of Theorem 1.15] For this we need as in the case of operators on closed manifolds, the cut-off integral $\int_{\mathbb{R}^{n}} \operatorname{tr}_{x}^{\tau} \sigma(x, \xi) d \xi$ of a local symbol $\sigma$ of a classical operator, which is defined in the same way as in (19), only replacing the fibrewise trace by $\operatorname{tr}^{\tau}$.

Proposition 3.11. For any holomorphic family $A(z) \in \Psi(M, \mathcal{E})$ of classical operators parametrised by $\mathbb{C}$, with local symbols $\sigma(z)$ and holomorphic order $-q z+a$ for some positive $q$ and some real number a, 
1. the meromorphic map $z \mapsto \operatorname{TR}_{x}^{\tau}(A(z)):=\int_{\mathbb{R}^{n}} \operatorname{tr}^{\tau}(\sigma(z)(x, \xi)) d \xi$ integrates over $M$ to the map

$$
z \mapsto \operatorname{TR}^{\tau}(A(z)):=\int_{M} \operatorname{TR}_{x}^{\tau}(A(z)) d x
$$

which is meromorphic with simple poles $d_{j}:=\frac{a+n-j}{q}, j \in \mathbb{Z}_{\geq 0}$.

2. 20] The complex residue at the point $d_{j}$ is given by:

$$
\operatorname{Res}_{z=d_{j}} \operatorname{TR}^{\tau}(A(z))=\frac{1}{q} \operatorname{Res}^{\tau}\left(A\left(d_{j}\right)\right) .
$$

3. 29. If $A\left(d_{j}\right)$ lies in $\Theta(M, E)$ i.e., if it is a differential operator, then $A^{\prime}\left(d_{j}\right)$ which need not be a classical pseudodifferential operator, nevertheless has a well defined Wodzicki residue

$$
\operatorname{Res}^{\tau}\left(A^{\prime}\left(d_{j}\right)\right):=\int_{M} \operatorname{res}_{x}^{\tau}\left(A^{\prime}\left(d_{j}\right)\right) d x
$$

where

$$
\operatorname{res}_{x}^{\tau}\left(A^{\prime}\left(d_{j}\right)\right):=\int_{|\xi|_{x}=1} \operatorname{tr}^{\tau}\left(\sigma_{-n}\left(A^{\prime}\left(d_{j}\right)\right)\right)(x, \xi) d_{S} \xi
$$

at the pole $d_{j}$ and we have

$$
\mathrm{fp}_{z=d_{j}} \operatorname{TR}^{\tau}(A(z))=\frac{1}{q} \operatorname{Res}^{\tau}\left(A^{\prime}\left(d_{j}\right)\right) .
$$

As in Theorem 2.6 we deduce the asymptotic expansion of the inverse Mellin transform of traces $\mathrm{TR}^{\tau}(A(z))$ of holomorphic families $A(z)$. Substituting $\mathrm{TR}^{\tau}$ to TR in Theorem 2.6 tells us that, if $f$ is continuous on ]0, $+\infty[$ with Mellin transform $z \mapsto \mathrm{TR}^{\tau}(A(z))$ for some holomorphic family $A(z) \in \Psi(M, \mathcal{E})$ of affine order $\alpha(z)=a-q z$ with $q$ some positive real number, then $f$ admits an asymptotic expansion at 0 given by

$$
f(t)=\frac{1}{q} \sum_{j \geq 0} a_{j} t^{-d_{j}}+O\left(t^{-\gamma}\right),
$$

with

$$
a_{j}=-\frac{1}{q} \operatorname{Res}^{\tau}\left(A\left(d_{j}\right)\right) \quad \text { for } \quad d_{j}>0,
$$

and constant term

$$
a_{j}=-\frac{1}{q} \operatorname{Res}^{\tau}\left(A^{\prime}(0)\right) \quad \text { for } \quad d_{j}=0 .
$$

Theorem 1.17 extends in a straightforward manner.

Theorem 3.12. Given a weight $Q \in \Psi(M, \mathcal{E})$ of order $q \in \mathbb{R}_{+}$and any operator $A \in \Psi(M, \mathcal{E})$ (so not necessarily admissible) of order $a \in \mathbb{R}$, the map

$$
z \mapsto \zeta^{\tau}(A, Q)(z):=\operatorname{TR}^{\tau}\left(A Q^{-z}\right)
$$

is holomorphic on the half plane $\Re(z)>\frac{n+a}{q}$ and defines a meromorphic map on $\mathbb{C}$ called the $\zeta^{\tau}$-regularised trace of $A$ with respect to the weight $Q$, with poles 
at $d_{j}=\frac{a+n-j}{q}, \quad j \in \mathbb{Z}_{\geq 0}$. The complex residue at such a pole is related to the Wodzicki $\tau$-residue of $A Q^{-d_{j}}$ by

$$
\operatorname{Res}_{z=d_{j}} \zeta^{\tau}(A, Q)(z)=\frac{1}{q} \operatorname{Res}^{\tau}\left(A Q^{-d_{j}}\right)
$$

For any differential operator $A \in \Theta(M, \mathcal{E})$, the $n$-form $\operatorname{res}_{x}^{\tau}(A \log Q) d x$ defines a global density on $M$ which integrates to the extended Wodzicki $\tau$-residue of $A \log Q$. The $\zeta^{\tau}$-regularised trace $\zeta^{\tau}(A, Q)(z)$ is holomorphic at zero and we have

$$
\zeta^{\tau}(A, Q)(0)=\lim _{z \rightarrow 0} \zeta^{\tau}(A, Q)(z)=-\frac{1}{q} \operatorname{Res}^{\tau}(A \log Q) .
$$

Remark 3.13. Let $A, \Delta$ be differential operators in $\Theta(M, \mathcal{E})$ with $\Delta$ admissible. Then exactly as in Proposition 1.8 one can prove that the pointwise extended Wodzicki residue $\operatorname{res}_{x}^{\tau}(A \log \Delta)$ is an algebraic expression in the coefficients of $A$ and in the $x$-jets of the coefficients of $\Delta$ at that point.

\subsection{The $\tau$-index as an extended $\tau$-residue}

The above constructions extend to $\mathbb{Z}_{2}$-graded vector bundles. Let $\mathcal{E}=\mathcal{E}_{+} \oplus \mathcal{E}_{-}$be a $\mathbb{Z}_{2}$-graded bundle of finite type $\mathcal{A}$-Hilbert modules over $M$ and let $D_{ \pm}: C^{\infty}\left(M, \mathcal{E}_{ \pm}\right)$ $\longrightarrow C^{\infty}\left(M, \mathcal{E}_{\mp}\right)$ be two elliptic differential operators of positive order $d$. We assume that the operators $D_{+}$and $D_{-}$are formally adjoint to each other which we write $D_{-}=D_{+}^{*}$. Hence

$$
D:=\left[\begin{array}{cc}
0 & D_{-} \\
D_{+} & 0
\end{array}\right]
$$

is essentially selfadjoint. Let $\Delta:=D^{2}=\left[\begin{array}{cc}D_{-} D_{+} & 0 \\ 0 & D_{+} D_{-}\end{array}\right]=\Delta_{+} \oplus \Delta_{-}$. The ellipticity of $D$ implies that the projection $\pi_{\Delta}$ onto $\operatorname{Ker} \Delta=\operatorname{Ker} D$ is a smoothing operator of finite $\tau$-rank 3, 34. Therefore one defines the $\tau$-dimension of the $\mathcal{A}$-Hilbert modules $\operatorname{Ker} D_{ \pm}$as

$$
\operatorname{dim}_{\tau}\left(\operatorname{Ker} D_{ \pm}\right):=\tau\left(\pi_{\Delta_{ \pm}}\right) \in \mathbb{R}
$$

and the difference

$$
\operatorname{ind}^{\tau} D_{+}:=\operatorname{dim}_{\tau}\left(\operatorname{Ker} D_{+}\right)-\operatorname{dim}_{\tau}\left(\operatorname{Ker} D_{-}\right) \in \mathbb{R}
$$

is called the $\tau$-index of the operator $D$. In the case $\mathcal{A}=\mathbb{C}$ this is the usual definition of the Fredholm index of the operator.

Remark 3.14. There exist smoothing perturbations $R$ of the nonnegative selfadjoint operator $D^{2}$ such that $D^{2}+R$ is invertible. Since we are in a von Neumann algebraic setting, this follows for example from [22, Proposition 2.10], see also [23.

Therefore there exist operators $R, R^{\prime}$ such that $D_{-} D_{+}+R$ and $D_{+} D_{-}+R^{\prime}$ are elliptic and nonnegative and hence so are their leading symbols nonnegative. Thus $\Delta, \Delta_{+}, \Delta_{-}$define admissible operators with spectral cut $\pi$.

Consequently, we can define the pointwise extended super $\tau$-residue of $\log \Delta$ as the difference of the pointwise extended residues of $\log \Delta_{+}$and $\log \Delta_{-}$

$$
\operatorname{sres}_{x}^{\tau}(\log \Delta)(x):=\operatorname{res}_{x}^{\tau}\left(\log \left(\Delta_{+}+R^{\prime}\right)\right)-\operatorname{res}_{x}^{\tau}\left(\log \left(\Delta_{-}+R\right)\right) .
$$

It can be integrated over $M$ to build the extended super $\tau$-residue

$$
\operatorname{sRes}^{\tau}(\log \Delta):=\frac{1}{(2 \pi)^{n}} \int_{M} \operatorname{sres}_{x}^{\tau}(\log \Delta)(x) d x .
$$


Corollary 3.15. The $\tau$-index of $D_{+}$is a local expression proportional to the extended Wodzicki (super) $\tau$-residue of the logarithm of $\Delta$

$$
\operatorname{ind}^{\tau}\left(D_{+}\right)=-\frac{1}{2 d} \operatorname{sRes}^{\tau}(\log (\Delta)) .
$$

Proof. The McKean-Singer formula combined with a Mellin transform yields for any positive real number $t$ and for any complex number $z$

$$
\begin{aligned}
\operatorname{ind}^{\tau}\left(D_{+}\right) & =\operatorname{Tr}^{\tau}\left(e^{-t\left(\Delta_{+}+\pi_{\Delta_{+}}\right)}\right)-\operatorname{Tr}^{\tau}\left(e^{-t\left(\Delta_{-}+\pi_{\Delta_{-}}\right)}\right) \\
& =\zeta_{\Delta_{+}+\pi_{\Delta_{+}}}^{\tau}(z)-\zeta_{\Delta_{-}+\pi_{\Delta_{-}}}(z) \\
& =-\frac{1}{2 d}\left(\operatorname{Res}^{\tau}\left(\log \left(\Delta_{+}+\pi_{\Delta_{+}}\right)\right)-\operatorname{Res}^{\tau}\left(\log \left(\Delta_{-}+\pi_{\Delta_{-}}\right)\right)\right) \\
& =-\frac{1}{2 d} \operatorname{Res}^{\tau}(\log \Delta) .
\end{aligned}
$$

\subsection{The extended residue for locally equivalent operators and Atiyah's $L^{2}$-index theorem}

In the following, denote by $(U ; \mathcal{E}, \mathcal{F})$ a triple where $U$ is a manifold and $\mathcal{E}, \mathcal{F}$ are bundles of finitely generated projective $\mathcal{A}$-Hilbert modules over $U$. Morphisms between these objects are of the form $\alpha=(f ; r, s):\left(U^{\prime} ; \mathcal{E}^{\prime}, \mathcal{F}^{\prime}\right) \rightarrow(X ; \mathcal{E}, \mathcal{F})$ where $f: U^{\prime} \rightarrow U$ is an open embedding, $r \in \operatorname{Hom}\left(\mathcal{E}^{\prime}, f^{*} \mathcal{E}\right), s \in \operatorname{Hom}\left(f^{*} \mathcal{F}, \mathcal{F}^{\prime}\right)$.

Given any linear map $L: C_{0}^{\infty}(U, \mathcal{E}) \rightarrow C^{\infty}(U, \mathcal{F})$, a morphism $\alpha:\left(U^{\prime} ; \mathcal{E}^{\prime}, \mathcal{F}^{\prime}\right) \rightarrow$ $(U ; \mathcal{E}, \mathcal{F})$ defines a map $\alpha^{\sharp} L: C_{0}^{\infty}\left(U^{\prime}, \mathcal{E}^{\prime}\right) \rightarrow C^{\infty}\left(U^{\prime}, \mathcal{F}^{\prime}\right)$ that makes the following diagram commute

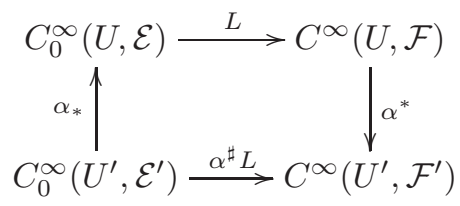

where $\alpha^{*}$ denotes the composition of $s$ with the map induced by the pullback, and $\alpha_{*}$ denotes the composition of $r$ with the push-forward.

Definition 3.16. Let $M, M^{\prime}$ be two manifolds and $A \in \Theta(M, \mathcal{E}), A^{\prime} \in \Theta\left(M^{\prime}, \mathcal{E}^{\prime}\right)$ be differential operators acting on the sections of $\mathcal{A}$-Hilbert modules bundles $\mathcal{E}, \mathcal{E}^{\prime}$ over $M$ and $M^{\prime}$ respectively. The operators $A$ and $A^{\prime}$ are said to be locally equivalent if

- there exists a local diffeomorphism $\phi: M^{\prime} \rightarrow M$ meaning that for any $x^{\prime}$ in $M^{\prime}$ there is a neighborhood $U^{\prime}$ of $x^{\prime}$ such that $U=\phi\left(U^{\prime}\right)$ is open and $\phi_{U^{\prime}}^{U}: U^{\prime} \rightarrow U$ is a diffeomorphism

- correspondingly, there are morphisms $\alpha=(f ; r, s):\left(U^{\prime}, \mathcal{E}_{\mid U^{\prime}}^{\prime}, \mathcal{E}_{\mid U^{\prime}}^{\prime}\right) \rightarrow\left(U, \mathcal{E}_{\mid U}, \mathcal{E}_{\mid U}\right)$ with $f=\phi_{U^{\prime}}^{U}$, and with $r, s$ isomorphisms such that

$$
A_{U^{\prime}}^{\prime}=\alpha^{\sharp}\left(A_{U}\right)
$$

where we have denoted by $A_{U^{\prime}}^{\prime}$ the restriction of $A^{\prime}$ to the open set $U^{\prime}$. 
Example 3.17. (Twists by flat bundles of Hilbert modules) Let $M$ be closed, $E \rightarrow M$ be a vector bundle, and $B \in \Theta(M, E)$ be a differential operator. Let $\mathcal{F} \rightarrow$ $M$ be a flat bundle of finitely generated projective $\mathcal{A}$-Hilbert modules, endowed with a flat connection $\nabla_{\mathcal{F}}$. Denote by $\mathcal{W}$ the fibre. Let $\underline{\mathcal{W}}=M \times \mathcal{W}$ denote the trivial bundle with fibre $\mathcal{W}$. Because $B$ is differential, one can consider on the one hand $B_{\underline{\mathcal{W}}}$ to be the trivial extension of $B$ to $E \otimes \underline{\mathcal{W}}$ and on the other hand $B_{\mathcal{F}}$ the operator $B$ twisted by the flat connection on $\mathcal{F}$. Then $A=B_{\underline{\mathcal{W}}}$ on $\mathcal{E}^{\prime}=E \otimes \underline{\mathcal{W}}$ and $A^{\prime}=B_{\mathcal{F}}$ on $\mathcal{E}^{\prime}=E \otimes \mathcal{F}$ are locally equivalent by taking $\pi$ the identity map on $M$, and the local morphisms given by local trivialisations of $\mathcal{F}$ which are parallel with respect to $\nabla_{\mathcal{F}}$.

Example 3.18. As a particular case of the above (with $\mathcal{W}=\ell^{2}(\Gamma)$ ), let $\mathcal{H}=$ $\widetilde{M} \times_{\Gamma} \ell^{2}(\Gamma)$ be the bundle defined in Example 3.4 and $\underline{\ell^{2} \Gamma}=M \times \ell^{2}(\Gamma)$. If $A \in \Theta(M, E)$ is a differential operator, $A_{\mathcal{H}}$ is locally equivalent to $A_{\underline{\ell^{2} \Gamma}}$.

Example 3.19. Specialising to the torus and using the notations of Example 3.5, for any differential operator $A$ on $\mathbb{T}^{n}$, the local equivalence between $A_{\mathcal{H}}$ and $A_{\underline{\ell^{2} \Gamma}}$ translates to the well-known local equivalence of the lifted operator $\pi^{\sharp} A$ (with a slight abuse of notation) on $\mathbb{R}^{n}$ with $A$.

Proposition 3.20. Let $A, A^{\prime}$ and $B, B^{\prime}$ be pairs of locally equivalent differential operators in the sense of Definition 3.16. Moreover assume that $B, B^{\prime}$ are admissible. Then with the notation of Definition 3.16

$$
\begin{aligned}
f^{*}\left(\operatorname{res}_{x}^{\tau}(A \log B) d x\right) & =\operatorname{res}_{x^{\prime}}^{\tau}\left(A^{\prime} \log \left(B^{\prime}\right)\right) d x^{\prime}, \\
f^{*}\left(\operatorname{res}_{x}^{\tau}\left(A B^{\alpha}\right) d x\right) & =\operatorname{res}_{x^{\prime}}^{\tau}\left(A^{\prime}\left(B^{\prime}\right)^{\alpha}\right) d x^{\prime}, \alpha \in \mathbb{R}
\end{aligned}
$$

for any point $x^{\prime}$ and any local diffeomorphism $f=\phi_{U^{\prime}}^{U}: U^{\prime} \rightarrow U$ on an open subset $U^{\prime}$ containing $x^{\prime}$, which when integrated over $M^{\prime}$ yields

$$
\begin{gathered}
\operatorname{Res}^{\tau}(A \log B)=\operatorname{Res}^{\tau}\left(A^{\prime} \log \left(B^{\prime}\right)\right) \\
\operatorname{Res}^{\tau}\left(A B^{\alpha}\right)=\operatorname{Res}^{\tau}\left(A^{\prime}\left(B^{\prime}\right)^{\alpha}\right), \quad \alpha \in \mathbb{R}
\end{gathered}
$$

Proof. Since $B$ and $B^{\prime}$ are admissible, there are smoothing operators $R, R^{\prime}$ such that $Q=B+R$ and $Q=B^{\prime}+R^{\prime}$ are weights. From Theorem 3.12 applied to the operator $A$ in $\Theta(M, \mathcal{E})$, we know that the $n$-form $x \mapsto \operatorname{res}_{x}^{\tau}(A \log B) d x$ defines a global density so it transforms covariantly under coordinate transformations. Thus, the pull-back of this density by the local diffeomorphism $f=\phi_{U^{\prime}}^{U}: U^{\prime} \rightarrow U$ reads

$$
f^{*}\left(\operatorname{res}_{x}^{\tau}(A \log B) d x\right)=\operatorname{res}_{x^{\prime}}^{\tau}\left(f^{\sharp} A \log f^{\sharp} B\right) d x^{\prime},
$$

where $x^{\prime}=f^{-1}(x)$. Now $A^{\prime}=f^{\sharp} A$ and $B^{\prime}=f^{\sharp} B$, so we get the result.

Specialising to (essentially) selfadjoint elliptic differential operators provides an alternative proof of Atiyah's $L^{2}$-index theorem in the Hilbert module formulation.

Corollary 3.21. (Atiyah's $L^{2}$-index theorem) Let $D$ be an essentially selfadjoint differential operator of positive order $d$ acting on a $\mathbb{Z}_{2}$-graded vector bundle $E^{+} \oplus$ $E^{-} \rightarrow M$, and assume $D$ is odd with respect to the grading, i.e. $D:=\left[\begin{array}{cc}0 & D_{-} \\ D_{+} & 0\end{array}\right]$. Let $D_{\mathcal{H}}:=\left[\begin{array}{cc}0 & D_{\mathcal{H},-} \\ D_{\mathcal{H},+} & 0\end{array}\right]$ be the twisted operator defined in Example 3.18 acting on the bundle of $\mathcal{N} \Gamma$-Hilbert modules $E \otimes \mathcal{H}$. Then

$$
\operatorname{ind}^{\tau}\left(D_{\mathcal{H},+}\right)=\operatorname{ind}\left(D_{+}\right) .
$$


Proof. Recall that by Example 3.17 the operator $D_{\mathcal{H}}^{2}$ is locally equivalent to the trivial extension $D_{\underline{\ell^{2} \Gamma}}$ acting on $\underline{\ell^{2} \Gamma}=M \times \ell^{2}(\Gamma)$. Using Remark 3.14, $D_{\mathcal{H}}^{2}$ is admissible (with $\pi$ as a spectral cut), so we can apply Proposition 3.20 which yields 10

$$
\operatorname{sRes}^{\tau}\left(\log \left(D_{\mathcal{H}}^{2}\right)\right)=\operatorname{sRes}^{\tau}\left(\log \left(D_{\underline{\ell^{2} \Gamma}}^{2}\right)\right) .
$$

By formula (57)

$$
\operatorname{ind}^{\tau}\left(D_{\mathcal{H},+}\right)=-\frac{1}{2 d} \operatorname{sRes}^{\tau}\left(\log \left(D_{\mathcal{H}}^{2}\right)\right)
$$

Analogously,

$$
\operatorname{ind}\left(D_{+}\right)=\operatorname{ind}^{\tau}\left(D_{\underline{\ell^{2} \Gamma},+}\right)=-\frac{1}{2 d} \operatorname{ses}^{\tau}\left(\log \left(D_{\underline{\ell^{2} \Gamma}}^{2}\right)\right)
$$

where we have used that $\operatorname{ind}^{\tau}\left(D_{\underline{\ell^{2}} \Gamma,+}\right)=\operatorname{ind}\left(D_{+}\right)$, so that the equality follows.

Remark 3.22. This is a self-contained pseudodifferential proof of Atiyah's result, which relies on the locality of the Wodzicki residue. It is very similar in spirit to John Roe's proof on coverings 31 .

Let $D$ be an essentially selfadjoint differential operator acting on a vector bundle $E \rightarrow M$. Let $D_{\mathcal{H}}$ be the twisted operator defined in Example 3.18.

Since $D_{\mathcal{H}}^{2}$ is admissible (see Remark 3.14), the operator $Q_{\mathcal{H}}:=D_{\mathcal{H}}^{2}+R$ defines a weight. Let us consider for any positive $t$ the associated heat-operator $e^{-t Q_{\mathcal{H}}}$. The corresponding heat-kernel $\tau$-trace $K_{t}^{\tau}\left(Q_{\mathcal{H}}\right)(x, x)$ at a point $x$ is defined by

$$
\operatorname{Tr}^{\tau}\left(\phi e^{-t Q_{\mathcal{H}}}\right):=\int_{M} \phi(x) K_{t}^{\tau}\left(Q_{\mathcal{H}}\right)(x, x) d x \quad \forall \phi \in C^{\infty}(M) .
$$

Applying as in Section 2 an inverse Mellin transform to the holomorphic families $A(z)=\phi Q_{\mathcal{H}}^{-z}$ and using Proposition 3.20 we find that

$$
\begin{aligned}
& K_{t}^{\tau}\left(D_{\mathcal{H}}^{2}\right)(x, x) \\
\sim & -\frac{(4 \pi)^{\frac{n}{2}}}{2 \sqrt{\operatorname{det} g}(x)}\left[\operatorname{res}_{x}^{\tau}\left(\log D_{\mathcal{H}}^{2}\right) \delta_{\frac{n}{2}-\left[\frac{n}{2}\right]}\right. \\
+ & \left.\sum_{k \in\left[0, \frac{n}{2}[\cap \mathbb{Z}\right.} \Gamma\left(\frac{n}{2}-k\right) \operatorname{res}_{x}^{\tau}\left(\left(D_{\mathcal{H}}^{2}\right)^{k-\frac{n}{2}}\right) t^{k-\frac{n}{2}}\right] \\
\sim & -\frac{(4 \pi)^{\frac{n}{2}}}{2 \sqrt{\operatorname{det} g}(x)}\left[\operatorname{res}_{x}\left(\log D^{2}\right) \delta_{\frac{n}{2}-\left[\frac{n}{2}\right]}\right. \\
+ & \left.\sum_{k \in\left[0, \frac{n}{2}[n \mathbb{Z}\right.} \Gamma\left(\frac{n}{2}-k\right) \operatorname{res}_{x}\left(\left(D^{2}\right)^{k-\frac{n}{2}}\right) t^{k-\frac{n}{2}}\right] .
\end{aligned}
$$

Remark 3.23. It follows from the Duhamel formula 4 that the time zero asymptotics of $K_{t}^{\tau}\left(D_{\mathcal{H}}^{2}\right)(x, x)$ coincide with that of $K_{t}^{\tau}\left(D_{\mathcal{H}}^{2}+R\right)(x, x)$. This is here confirmed by the fact that the residues involved in the asymptotics are invariant under perturbation by the smoothing operator $R$.

${ }^{10}$ Prop. 3.20 easily extends to the $\mathbb{Z}_{2}$-graded case replacing the $\tau$-residue by the super $\tau$-residue. 


\section{The scalar curvature on the noncommutative two-torus}

We want to define the scalar curvature on the noncommutative two-torus by means of a Wodzicki residue in analogy to the formula (40) established for Riemannian surfaces. We need a noncommutative analogue of Theorem 1.17 on the noncommutative torus $\mathbb{T}_{\theta}^{n}$. Let us briefly recall the results of [24] we need for that purpose.

\subsection{Pseudodifferential operators on the noncommutative torus}

Let $\theta$ be a symmetric $n \times n$ real matrix. The noncommutative deformation $\mathbb{T}_{\theta}^{n}$ of the commutative torus $\mathbb{T}^{n} \sim \mathbb{R}^{n} / \mathbb{Z}^{n}$ is encoded in the $C^{*}$-algebra $A_{\theta}$. An element $a \in A_{\theta}$ decomposes as the convergent series $a=\sum_{k \in \mathbb{Z}^{n}} a_{k} U_{k}$ where the $\left(U_{k}\right)$ are unitaries in $A_{\theta}$ that satisfy $U_{0}=1$ and

$$
U_{k} U_{l}=e^{-2 \pi i\langle k, \theta l\rangle} U_{l} U_{k} .
$$

Let $\mathcal{A}_{\theta}$ denote the algebra consisting of series of the form $\sum_{k \in \mathbb{Z}^{n}} a_{k} U_{k}$, where the sequence $\left(a_{k}\right)_{k} \in \mathcal{S}\left(\mathbb{Z}^{n}\right)$, the vector space of sequences $\left(a_{k}\right)_{k}$ that decay faster than the inverse of any polynomial in $k$. We shall also need the linear form $\mathbf{t}$ on $A_{\theta}$ which to an element $a=\sum_{k \in \mathbb{Z}^{n}} a_{k} U_{k}$ assigns the scalar term $a_{0}$, and the Laplace operator $\boldsymbol{\Delta}=\sum_{j} \delta_{j}^{2}$ defined in [24, Example 3.13] acting on $A_{\theta}$ with $\delta_{j}\left(\sum_{k \in \mathbb{Z}^{n}} a_{k} U_{k}\right)=\sum_{k \in \mathbb{Z}^{n}} k_{j} a_{k} U_{k}$.

We refer to 24 for the construction of the corresponding algebra $\Psi\left(\mathbb{T}_{\theta}^{n}\right)$ of classical toroidal pseudodifferential operators [24, Paragraph 3.2] on $\mathbb{T}_{\theta}^{n}$. When $\theta=0$, the noncommutative torus $\mathbb{T}_{\theta}^{n}$ coincides with $\mathbb{T}^{n}, \mathcal{A}_{\theta}$ with $C^{\infty}\left(\mathbb{T}^{n}\right)$ and $\Psi\left(\mathbb{T}_{\theta}^{n}\right)$ with the algebra $\Psi\left(\mathbb{T}^{n}\right)$ of classical pseudodifferential operators on the closed manifold $\mathbb{T}^{n}$ considered in the first section.

\subsection{Holomorphic families of operators on the noncommu- tative torus}

In 24] we defined holomorphic families in $\Psi\left(\mathbb{T}_{\theta}^{n}\right)$ and extended the canonical trace to such families by

$$
\operatorname{TR}_{\theta}(A(z)):=\sum_{\mathbb{Z}^{n}} \mathbf{t}\left(\mathrm{Op}_{\theta}^{-1}(A(z))\right)
$$

where $\mathrm{Op}_{\theta}$ is the one to one map which takes a toroidal symbol to a toroidal operator. As seen in [24, Proposition 6.2], the Wodzicki residue $\operatorname{Res}_{\theta}$ on $\mathcal{A}_{\theta}$, is a noncommutative analogue of the classical Wodzicki residue and (up to a multiplicative factor) it is the only continuous linear form on $\Psi\left(\mathbb{T}_{\theta}^{n}\right)$ vanishing on smoothing operators [14] (see also 24 for a slightly different characterisation which does not require continuity). In contrast to this, the canonical trace $\mathrm{TR}_{\theta}$ is (up to a multiplicative factor) the only linear form on non integer operators in $\Psi\left(\mathbb{T}_{\theta}^{n}\right)$ whose restriction to trace-class operators is continuous 24 .

Theorem 4.1. For any holomorphic family $A(z) \in \Psi\left(\mathbb{T}_{\theta}^{n}\right)$ of classical operators parametrised by $\mathbb{C}$ with holomorphic order $-q z+$ a for some positive $q$ and some real number a,

1. the map $z \mapsto \operatorname{TR}_{\theta}(A(z))$ is meromorphic with simple poles $d_{j}:=\frac{a+n-j}{q}$, $j \in \mathbb{Z}_{\geq 0}$ 
2. the complex residue at the point $d_{j}$ is given by:

$$
\operatorname{Res}_{z=d_{j}} \operatorname{TR}_{\theta}(A(z))=\frac{1}{q} \operatorname{Res}_{\theta}\left(A\left(d_{j}\right)\right) .
$$

3. If $A\left(d_{j}\right)$ is a differential operator, then $A^{\prime}\left(d_{j}\right)$ has a well defined Wodzicki residue $\operatorname{Res}_{\theta}\left(A^{\prime}\left(d_{j}\right)\right)$ and we have

$$
\mathrm{fp}_{z=d_{j}} \operatorname{TR}(A(z))=\frac{1}{q} \operatorname{Res}_{\theta}\left(A^{\prime}\left(d_{j}\right)\right) .
$$

It was shown in 24 how one can define via a Cauchy formula the logarithm [24. Paragraph 7] $\log (\boldsymbol{\Delta})$ of the Laplace operator and a noncommutative analogue $\zeta_{\theta}(A, Q)$ [24, Paragraph 7] of the $Q$-regularised $\zeta$-trace of $A$ with $Q:=1+\Delta$. Applying Theorem 4.1 to the holomorphic family $A(z)=A Q^{-z}$ yields the following extension of Theorem 1.17 to the noncommutative torus.

Theorem 4.2. Let $Q:=1+\Delta$ and let $A$ be a differential operator in $\Psi\left(\mathbb{T}_{\theta}^{n}\right)$. Then

1. the $\zeta_{\theta}$-regularised trace $\zeta_{\theta}(A, Q)$ of $A$ is holomorphic at zero,

2. the residue $\operatorname{Res}_{\theta}$ extends to $A \log Q$ and we have

$$
\zeta_{\theta}(A, Q)(0)=-\frac{1}{q} \operatorname{Res}_{\theta}(A \log Q) .
$$

\subsection{The scalar curvature as an extended Wodzicki residue}

We now want to define the scalar curvature on $\mathbb{T}_{\theta}^{2}$ by means of a noncommutative analogue of (40). We work on a conformal deformation of the complexified two torus using the notational conventions of [12.

Let $\tau=\tau_{1}+i \tau_{2}$ with $\tau_{1}, \tau_{2} \in \mathbb{R}$. Let $\partial=\delta_{1}+\bar{\tau} \delta_{2}$. Then $\partial^{*}=\delta_{1}+\tau \delta_{2}$. The operators $\partial_{\tau}$ and $\partial_{\tau}^{*}$ are the noncommutative counterparts of $-i\left(\partial_{x_{1}}+\bar{\tau} \partial_{x_{2}}\right)$ and $-i\left(\partial_{x_{1}}+\tau \partial_{x_{2}}\right)$ acting on $\mathcal{A}_{0}=C^{\infty}\left(\mathbb{T}^{2}\right)$. Let $h \in \mathcal{A}_{\theta}$ be selfadjoint and set $k=e^{\frac{h}{2}}$. Let $\mathcal{H}_{h}$ be the completion of $\mathcal{A}_{\theta}$ for the inner product

$$
\langle a, b\rangle_{h}=\mathbf{t}\left(b^{*} a k^{-2}\right)=\left\langle a k^{-1}, b k^{-1}\right\rangle_{0}=\left\langle R_{k^{-2}} a, b\right\rangle_{0}
$$

on $\mathcal{A}_{\theta}$ where $R_{b}: a \mapsto a b$ stand for the right multiplication by $b$. The map $R_{k}: a \mapsto a k$ induces an isometry $U: \mathcal{H}_{0} \longrightarrow \mathcal{H}_{h}$.

The analogue of the space of $(1,0)$-forms on the ordinary two-torus is defined to be the Hilbert space completion $\mathcal{H}_{0}^{(1,0)}$ of the space of finite sums $a \partial b, a, b \in \mathcal{A}_{\theta}$ for the inner product $\langle a, b\rangle_{0}$. We view $\partial$ as an unbounded operator $\partial_{h}: \mathcal{H}_{h} \longrightarrow \mathcal{H}_{0}^{(1,0)}$. Then

$$
\langle\partial a, b\rangle_{0}=\left\langle a, \partial^{*} b\right\rangle_{0}=\left\langle R_{k^{2}} a, \partial^{*} b\right\rangle_{h}=\left\langle a, R_{k^{2}} \partial^{*} b\right\rangle_{h} .
$$

Thus its formal adjoint is given by

$$
\partial_{h}^{*}=R_{k^{2}} \partial^{*} .
$$

We consider the operator

$$
D_{h}=\left[\begin{array}{cc}
0 & \partial_{h}^{*} \\
\partial_{h} & 0
\end{array}\right]=\left[\begin{array}{cc}
0 & R_{k^{2}} \partial^{*} \\
\partial & 0
\end{array}\right]
$$


acting on the $\mathbb{Z}_{2^{-}}$graded space $\widetilde{\mathcal{H}_{h}}:=\mathcal{H}_{h} \oplus \mathcal{H}_{0}^{(1,0)}$. Let

$$
\boldsymbol{\Delta}_{h}:=\partial_{h}^{*} \partial_{h}+\partial_{h} \partial_{h}^{*}=R_{k^{2}} \partial^{*} \partial+\partial R_{k^{2}} \partial^{*},
$$

which for $\tau_{i}$ and $h=0$ coincides with $\boldsymbol{\Delta}$.

Here is a corollary of Theorem 1.17 extended to this slightly more general framework.

Corollary 4.3. Let $Q:=\boldsymbol{\Delta}_{h}+\pi_{\boldsymbol{\Delta}_{h}}$, where $\pi_{\boldsymbol{\Delta}_{h}}$ is the orthogonal projection onto the kernel of $\boldsymbol{\Delta}$ and let $a \in \mathcal{A}_{\theta}$. Then

1. the $\zeta_{\theta}$-regularised trace $\zeta_{\theta}(a, Q)$ of a is holomorphic at zero,

2. the residue $\operatorname{Res}_{\theta}$ extends to $a \log Q$ and we have

$$
\zeta_{\theta}(a, Q)(0)=-\frac{1}{q} \operatorname{Res}_{\theta}(a \log Q) .
$$

Exactly as in the case of manifolds, if we specialise the holomorphic family to the case $A(z)=a Q^{-z}$, where $a$ is an element of the algebra of the noncommutative torus, using the same arguments, we get the following result:

Theorem 4.4. For any $a \in \mathcal{A}_{\theta}$ we have

$$
\begin{aligned}
& \operatorname{Tr}\left(a e^{-t \boldsymbol{\Delta}_{h}}\right) \sim_{t \rightarrow 0} \\
& -\frac{(4 \pi)^{\frac{n}{2}}}{2}\left[\operatorname{Res}\left(a \log \boldsymbol{\Delta}_{h}\right) \delta_{\frac{n}{2}-\left[\frac{n}{2}\right]}\right. \\
& \left.+\sum_{k \in\left[0, \frac{n}{2}[\cap \mathbb{Z}\right.} \Gamma\left(\frac{n}{2}-k\right) \operatorname{Res}\left(a \boldsymbol{\Delta}_{h}^{k-\frac{n}{2}}\right) t^{k-\frac{n}{2}}\right] .
\end{aligned}
$$

The scalar curvature being associated to the $a_{1}$ coefficient of the heat kernel expansion, Theorem 4.4 motivates the following definition:

Definition 4.5. The "scalar curvature" $\mathfrak{s}_{h}$ on the noncommutative two torus $\mathbb{T}_{\theta}^{2}$ associated with the "metric" determined by the conformal factor $h$ is defined as

$$
\left\langle\mathfrak{s}_{h}, a\right\rangle_{h}= \begin{cases}-6 \pi \operatorname{Res}_{\theta}\left(a \log \boldsymbol{\Delta}_{h}\right) & \text { if } n=2 \\ -\frac{3}{2}(4 \pi)^{\frac{n}{2}} \Gamma\left(\frac{n}{2}-k\right) \operatorname{Res}_{\theta}\left(a \boldsymbol{\Delta}_{h}^{k-\frac{n}{2}}\right) & \text { otherwise, }\end{cases}
$$

which compares with the definitions in [7, [8, 11, 12.

\section{Acknowledgment}

The last author thanks the organisers of the XXXIII Workshop on Geometric Methods in Physics for giving her the opportunity to present some of these results during the meeting.

\section{References}

[1] T. Ackermann, A note on the Wodzicki residue, J. Geometry and Physics 20 (1996) 404-406 
[2] M.-T. Benameur, T. Fack, Type II non-commutative geometry. I. Dixmier trace in von Neumann algebras, Adv. in Math. 199 (2006) 29-87

[3] D. Burghelea, L. Friedlander, T. Kappeler, P. McDonald, Analytic and Reidemeister torsion for representations in finite type Hilbert modules, Geometric and Functional Analysis 6, n. 5 (1996)

[4] N. Berline, E. Getzler, M. Vergne, Heat-kernels and Dirac operators, Grundlehren der math. Wiss. 298 Springer Verlag 1996

[5] A. Connes, A. Chamseddine, Inner fluctuations of the spectral action, J. Geom. Phys. 73 (2013) 222-234

[6] A. Connes and H. Moscovici, The local index formula in noncommutative geometry, Geom. Func. Anal. 5 (1995), 174-243

[7] A. Connes and H. Moscovici, Modular curvature for noncommutative two-tori, arXiv:1110.3500v1 [math.OA] (2011)

[8] A. Connes and P. Tretkhoff, The Gauss-Bonnet theorem for the noncommutative two torus, Noncommutative geometry, arithmetic and related topics, Eds. C. Consani and A. Connes, John Hopkins University Press (2011), 141-158

[9] P. Flajolet, X. Gourdon, P. Dumas, Mellin transforms and asymptotics: Harmonic sums, Theoretical Computer Science, 144(1-2): 3-58, 1995

[10] E. Freitag, R. Busam, Complex Analysis, Springer-Verlag Berlin Heidelberg, 2005

[11] F. Fathizadeh and M. Khalkhali, Scalar curvature for the noncommutative two torus, arXiv:1110.3511 (2011)

[12] F. Fathizadeh and M. Khalkhali, The Gauss-Bonnet theorem for noncommutative two tori with a general conformal structure, J. Noncommut. Geom. 6 (2012), no. 3, 457-480

[13] F. Fathizadeh and M. Khalkhali, Scalar curvature for noncommutative fourtori, arXiv:1301.6135 (2013)

[14] F. Fathizadeh and M.W. Wong, Noncommutative residues for pseudodifferential operators on the noncommuative two-torus, J. Pseudodiff. Oper. Appl. (2) (2011), 289-302

[15] P. Gilkey, Invariance theory, the heat equation and the Atiyah-Singer index theorem, Second Edition, Studies in advanced mathematics, 1995

[16] I.M. Gelfand, Generalized functions, Vol. 1 Academic Press, 1964

[17] V. Guillemin, Gauged Lagrangian distributions, Adv. Math. 102 (1993) 184201

[18] P. Jeanquartier, Transformation de Mellin et développements asymptotiques, L'Enseignement Mathématique 25 (1979)

[19] W. Kalau, M. Walze, Gravity, noncommutative geometry and the Wodzicki residue, J. Geometry and Physics 16 (1995) 327-344 
[20] M. Kontsevich, S. Vishik, Geometry of determinants of elliptic operators, Func. Anal. on the Eve of the XXI century, Vol I, Progress in Mathematics 131 (1994) 173-197; Determinants of elliptic pseudo-differential operators, Max Planck Preprint (1994)

[21] M. Lesch, On the noncommutative residue for pseudo-differential operators with log-polyhomogeneous symbols, Ann. Global Anal. Geom. 17 (1998) 151187

[22] E. Leichtnam, P. Piazza. Spectral sections and higher Atiyah-Patodi-Singer index theory on Galois coverings. Geom. Funct. Anal., 8 (1) 17-58, (1998)

[23] E. Leichtnam, P. Piazza. Dirac index classes and the non-commutative spectral flow. J. Funct. Anal., 200 (2) 348-400, (2003)

[24] C. Lévy, C. Neira-Jiménez, S. Paycha, The canonical trace and the noncommutative residue on the noncommutative torus, arXiv:1303.0241, to appear in Trans. Am. Math. Soc.

[25] J. Mickelsson, S. Paycha, Logarithmic residue density of a generalised Laplacian, J. Aust. Math. Soc. 90 (2001) 53-80

[26] H. McKean, I.M. Singer, Curvature and the eigenvalues of the Laplacian, J. Diff. Geom. 1 (1967) 43-69

[27] K. Okikiolu, The Campbell-Hausdorff theorem for elliptic operators and a related trace formula, Duke. Math. Journ. 79 (1995) 687-722

[28] K. Okikiolu, The multiplicative anomaly for determinants of elliptic operators, Duke Math. Journ. 79 (1995) 722-749

[29] S. Paycha, S. Scott, A Laurent expansion for regularised integrals of holomorphic symbols, Geom. Funct. Anal. 17 (2007) 491-536

[30] P. Piazza, T. Schick, Bordism, rho-invariants and the Baum-Connes conjecture, J. Noncommut. Geom. 1, (2007) 27-111

[31] J. Roe, Elliptic operators, topology and asymptotic methods, Second edition, 1998, Chapmann and Hall

[32] S. Rosenberg, The Laplacian on a Riemannian manifold, London Mathematical Society, Student Texts 31, Cambridge University Press, 1997

[33] M. Ruzhansky, V. Turunen, Pseudodifferential operators and symmetries, Background analysis and advanced topics. Pseudo-Differential Operators. Theory and Applications, 2. Birkhäuser Verlag, Basel, 2010

[34] T. Schick, $L^{2}$-index theorems, KK-theory, and connections, New York J. Math. 11 (2005), 387-443

[35] S. Scott, The residue determinant, Comm. Partial Differential Equations 30 no. 4-6 (2005), 483-507

[36] S. Scott, Traces and Determinants of Pseudodifferential Operators, Oxford Mathematical Monographs, 2010

[37] R.T. Seeley, Complex powers of an elliptic operator, Singular integrals, Proc. Symp. Pure Math., Chicago, Amer. Math. Soc., Providence (1966) 288-307 
[38] S. Vassout, Feuilletages et résidu non commutatif longitudinal, Thèse de Doctorat, Paris 6, 2001

[39] M. Wodzicki, Noncommutative residue. I. Fundamentals., Lecture Notes in Math., 1289, Springer, Berlin, 1987

Sara Azzali

Institut für Mathematik

Universität Potsdam

Am Neuen Palais, 10

14469 Potsdam, Germany

e-mail: azzali@uni-potsdam.de

Cyril Lévy

Département de mathématiques

Centre universitaire Jean-François Champollion

Place Verdun

81000 Albi, France

e-mail: cyril.olivier.levy@gmail.com

Carolina Neira-Jiménez

Departamento de Matemáticas

Universidad Nacional de Colombia

Carrera 30 \# 45-03

Bogotá, Colombia

e-mail: cneiraj@unal.edu.co

Sylvie Paycha

Institut für Mathematik

Universität Potsdam

Institut für Mathematik

Am Neuen Palais, 10

14469 Potsdam, Germany

e-mail: paycha@math.uni-potsdam.de 